\title{
WestVirginiaUniversity
}

THE RESEARCH REPOSITORY @ WVU

Graduate Theses, Dissertations, and Problem Reports

2020

\section{Image and Video-Based Autism Spectrum Disorder Detection via Deep Learning}

\author{
Mindi Ruan \\ mr0114@mix.wvu.edu
}

Follow this and additional works at: https://researchrepository.wvu.edu/etd

Part of the Artificial Intelligence and Robotics Commons, and the Cognitive Neuroscience Commons

\section{Recommended Citation}

Ruan, Mindi, "Image and Video-Based Autism Spectrum Disorder Detection via Deep Learning" (2020). Graduate Theses, Dissertations, and Problem Reports. 7818.

https://researchrepository.wvu.edu/etd/7818

This Thesis is protected by copyright and/or related rights. It has been brought to you by the The Research Repository @ WVU with permission from the rights-holder(s). You are free to use this Thesis in any way that is permitted by the copyright and related rights legislation that applies to your use. For other uses you must obtain permission from the rights-holder(s) directly, unless additional rights are indicated by a Creative Commons license in the record and/ or on the work itself. This Thesis has been accepted for inclusion in WVU Graduate Theses, Dissertations, and Problem Reports collection by an authorized administrator of The Research Repository @ WVU. For more information, please contact researchrepository@mail.wvu.edu. 


\title{
Image and Video-Based Autism Spectrum Disorder Detection via Deep Learning
}

\author{
Mindi Ruan \\ Thesis submitted to the \\ Benjamin M. Statler College of Engineering and Mineral Resources \\ at West Virginia University \\ in partial fulfillment of the requirements for the degree of \\ Master \\ in \\ Computer Science \\ Xin Li, Ph.D., Chair \\ Shuo Wang, Ph.D. \\ Donald A. Adjeroh, Ph.D.
}

Lane Department of Computer Science and Electrical Engineering

Morgantown, West Virginia

2020

Keywords: Deep learning, Autism Spectrum Disorder Diagnose, Skeleton-base action recognition, Adaptive graph, Photos, Videos

Copyright 2020 Mindi Ruan 


\begin{abstract}
Image and Video-Based Autism Spectrum Disorder Detection via Deep Learning

Mindi Ruan
\end{abstract}

People with Autism Spectrum Disorder (ASD) show atypical attention to social stimuli and aberrant gaze when viewing images of the physical world. However, it is unknown how they perceive the world from a first-person perspective. In this study, we used machine learning to classify photos taken in three different categories (people, indoors, and outdoors) as either having been taken by individuals with ASD or by peers without ASD. Our classifier effectively discriminated photos from all three categories but was particularly successful at classifying photos of people with $>80 \%$ accuracy. Importantly, the visualization of our model revealed critical features that led to successful discrimination and showed that our model adopted a strategy similar to that of ASD experts. Furthermore, for the first time, we showed that photos taken by individuals with ASD contained less salient objects, especially in the central visual field. Notably, our model outperformed the classification of these photos by ASD experts. Together, we demonstrate an effective and novel method that is capable of discerning photos taken by individuals with ASD and revealing aberrant visual attention in ASD from a unique first-person perspective. Our method may in turn provide an objective measure for evaluations of individuals with ASD.

People with ASD also show atypical behavior when they are doing the same action with peers without ASD. However, it is challenging to efficiently extract this feature from spatial and temporal information. In this study, we applied Graph Convolutional Network (GCN) to the 2D skeleton sequence to classify video recording the same action (brush teeth and wash face) as either from individuals with ASD or by peers without ASD. Furthermore, we adopted an adaptive graph mechanism that allows the model to learn a kernel flexibly and exclusively for each layer, which means the model can learn more useful and robust features. Our classifier can effectively reach $80 \%$ accuracy. Our method may play an important role in the evaluations of individuals with ASD. 


\section{Acknowledgments}

First, I would like to express my deepest appreciation to my committee chair and advisor Prof. Xin Li and co-advisor Prof. Shuo Wang for giving me invaluable guidance and advice throughout my master's study in the past two years. Their professionalism, expertise, and patience help me build up self-confidence and motivate me to find new insights on research and finally published my first paper in my life. This thesis would not be possible without their enormous effort and generous help. Their rigorous and enthusiastic research attitudes have always deeply guided and inspired me in my whole master's study. And I believe these experiences at West Virginia University will benefit me a lot in my future life and career.

I would like to extend my gratitude to Prof. Donald A. Adjeroh for giving me valuable suggestions for my master's study and helping me to improve this thesis. Also, I am thankful to all my lab mates, colleagues, faculty and staff members in Lane Department of Computer Science and Electrical Engineering, and all my friends, for their generous help and support during my master's study.

Finally, I would like to thank my family, especially my parents and my sister, who unconditionally love, support, and understand me. 


\section{Contents}

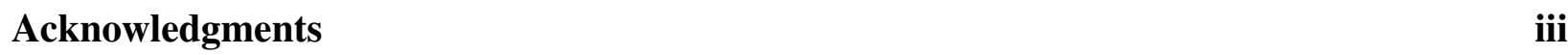

List of Figures

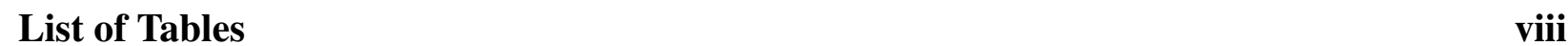

\begin{tabular}{ll}
\hline List of Abbreviations & ix
\end{tabular}

\begin{tabular}{lll|}
\hline 1 & Introduction & 1
\end{tabular}

1.1 Motivation . . . . . . . . . . . . . . . . . . . . . 1

$1.1 .1 \quad$ Analysis of $\mathrm{ASD}$ First-Person Perspective . . . . . . . . . . . . . . . 1

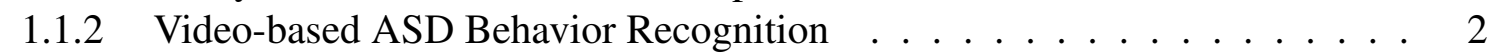

1.2 Problem Statement . . . . . . . . . . . . . . . . . . . . . . 3

$1.2 .1 \quad$ Analysis of ASD First-Person Perspective . . . . . . . . . . . . . . . . . . . 3

1.2.2 $\quad$ Video-based[ASD Behavior Recognition $\ldots \ldots \ldots \ldots$. . . . . . . . 4

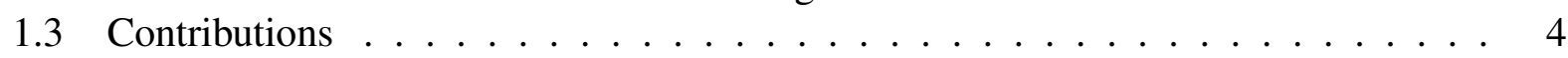

1.4 Dissertation Structure $\ldots \ldots \ldots \ldots \ldots$

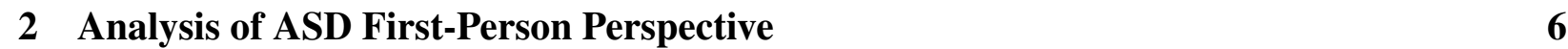

2.1 Related Work . . . . . . . . . . . . . . . . . . . . . 6

2.2 Dataset and Materials . . . . . . . . . . . . . . . . . . . . 7

2.2 .1 Participants . . . . . . . . . . . . . . . . . . 7

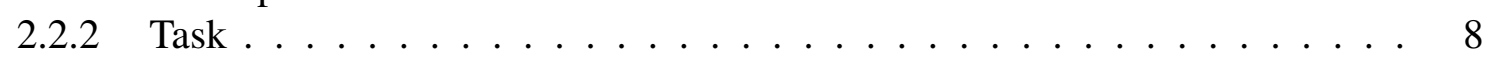

2.2 .3 Rating bylASDExperts . . . . . . . . . . . . . . . . 9

$2.2 .4 \quad$ First-Person Perspective Dataset . . . . . . . . . . . . . . . . . . . . . 9

2.3 Methods . . . . . . . . . . . . . . . . . . . . . 9

$2.3 .1 \quad$ Classification . . . . . . . . . . . . . . . . . . . . . . . . 9

2.3 .2 Receiver Operating Characteristic Curves . . . . . . . . . . . . . . . . 11

2.3 .3 Saliency Analysis . . . . . . . . . . . . . . . . . . 12

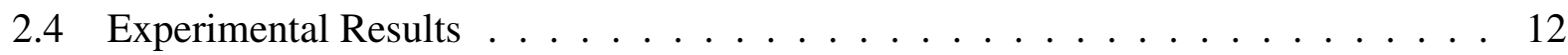

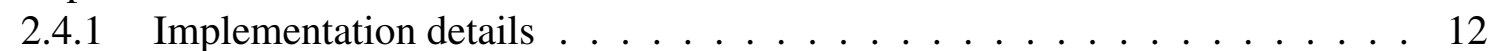

2.4 .2 Model Performance . . . . . . . . . . . . . . . . . . . . . . . 13

$2.4 .3 \quad$ Model Explanation Through Layer-Wise Relevance Propagation . . . . . . 14

2.4 .4 Saliency Analysis of Photos . . . . . . . . . . . . . . 16

2.4 .5 Comparison With Human Performance $\ldots \ldots \ldots$ 


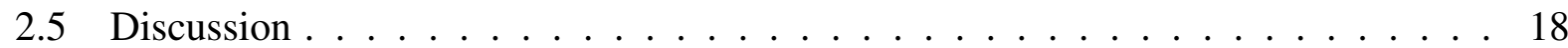

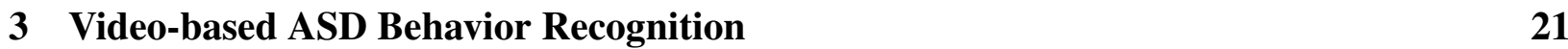

3.1 Related Work . . . . . . . . . . . . . . . . . . . . . . . 21

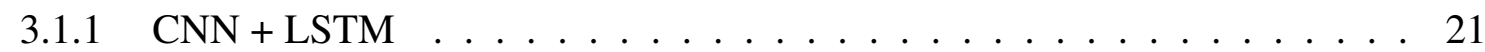

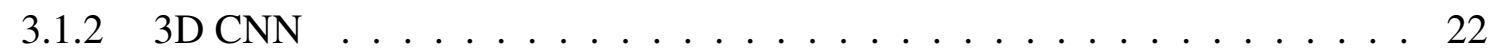

3.1 .3 Two Stream Network . . . . . . . . . . . . . . . . . . . . 23

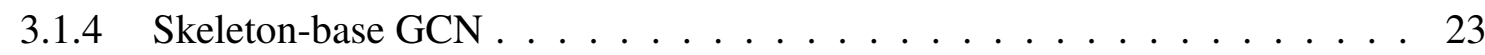

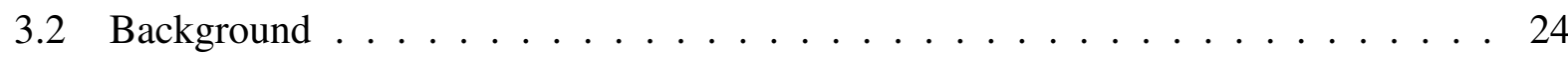

$3.2 .1 \quad$ Spatial-temporal graph . . . . . . . . . . . . . . . . . . 24

3.2.2 Human skeleton graph convolution . . . . . . . . . . . . . . . 24

3.3 Datasets and Data Preprocessing . . . . . . . . . . . . . . 25

3.3 .1 NTURGB+D . . . . . . . . . . . . . . . . . . . . . . . . . . . . . . . . . .

3.3 .2 ASD Demonstration Task Interview $\ldots \ldots \ldots \ldots$. . . . . . . . 25

3.4 Methods and Modules of Adaptive Graph $\ldots \ldots \ldots$. . . . . . . . . . . 27

3.4.1 Model Architecture . . . . . . . . . . . . . . . . . . . . . . . 27

3.4 .2 Adaptive Graph . . . . . . . . . . . . . . . . . . . . . . . . . 28

3.5 Experimental Results . . . . . . . . . . . . . . . . . . . . . . . 29

3.5 .1 Implementation Details . . . . . . . . . . . . . . . . . . . . 29

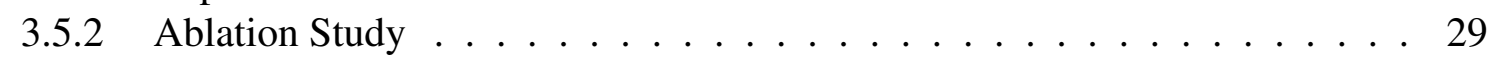

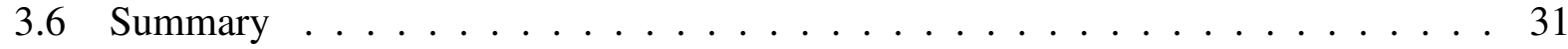

4 Conclusions and Future Work $\quad 32$

$4.1 \quad$ Analysis of ASD First-Person Perspective . . . . . . . . . . . . . . . . . 32

4.2 ASD Behavior Recognition $\ldots \ldots \ldots \ldots \ldots \ldots$

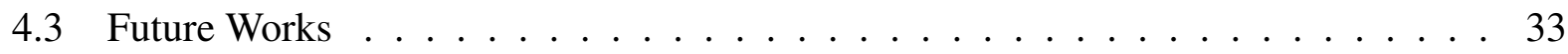

\begin{tabular}{ll}
\hline Bibliography & 35
\end{tabular}

\begin{tabular}{|l|l|}
\hline A & Supporting Information \\
\hline
\end{tabular} 


\section{List of Figures}

2.1 Model architecture and performance. (A) Model architecture. The input was a fixed-size $224 \times 224$ RGB image. The image was passed through a stack of convolutional layers, where the filters were used with a very small receptive field of $3 \times 3$. The convolution stride was fixed to 1 pixel; the spatial padding of convolutional layer input was such that the spatial resolution was preserved after convolution (i.e., the padding was 1-pixel for $3 \times 3$ convolutional layers). Spatial pooling was carried out by five max-pooling layers, which followed some of the convolutional layers. Max-pooling was performed over a $2 \times 2$ pixel window, with stride 2 . Three fully connected (FC) layers followed a stack of convolutional layers: the first two had 4096 channels each, the third performed a two-way[ASD classification and thus contained two channels. The final layer was the Softmax layer. All hidden layers were equipped with the rectification (ReLU) nonlinearity. (B) Model prediction accuracy. Our model could differentiate photos taken by those with ASD from those taken by controls in all conditions. Error bars denote \pm SEM across runs and circles show individual values. Asterisks indicate a significant difference in prediction accuracy between observed (magenta) versus permuted (gray) runs using unpaired t-test: $* * *: \mathrm{P}<0.001 . \ldots \ldots$

2.2 Layer-wise Relevance Propagation (LRP) explanation. (A-K) Photos that were classified as having been taken by participants with ASD (L-O) Photos that were classified as having been taken by controls. Red pixels positively contributed to the classification whereas blue pixels negatively contributed to the classification.

(J, K) Photos that were incorrectly classified as having been taken by participants with ASD. (N, O) Photos that were incorrectly classified as having been taken by controls. The cyan box in (F) illustrates where the subject of the photo was located. 15

2.3 Saliency analysis. (A-C) People Block. (D-F) Indoor Block. (G-I) Outdoor Block. (A, B, D, E, G, H) Example photos (left) with detected saliency maps (right). (A, D, G) Photos were taken by participants with ASD, (B, E, H) Photos taken by controls. (C, F, I) Average saliency value. Error bars denote \pm SEM across photos. Solid bars denote the central region and open bars denote the peripheral region. Asterisks indicate significant difference between $[\mathrm{ASD}$ and controls using two-tailed unpaired t-test: *: $\mathrm{P}<0.05$, **: $\mathrm{P}<0.01$, and ***: $\mathrm{P}<0.001$. Red: |ASD| Blue: controls. . . . . . . . . . . . . . . . . . . 16 
2.4 Our classifier model outperformed human $\mathrm{ASD}$ experts. (A, B) People Block. (C, D) Indoor Block. (E, F) Outdoor Block. (A, C, E) Receiver Operating Characteristic ( $\mathrm{ROC})$ curves. (B, D, F) The area under the $\mathrm{ROC}$ curve (Area Under the Curve (AUC) values). Error bars denote \pm SEM across runs and circles show individual values. Asterisks indicate a significant difference in performance between machine learning (purple) and ASD professionals (yellow) using paired t-test: $* * *$ : $\mathrm{P}<0.001 \ldots \ldots \ldots \ldots \ldots \ldots$

3.1 The main modalities in human action recognition area. (A) CNN+LSTM; (B) 3D CNN; (3) Two-Stream Network. $\mathrm{N}$ indicates for the total number of frames in a video, whereas K stands for a subset of neighboring frames of the video.. . . . . . 22

3.2 Illustration of the human skeleton graphs in NTU RGB+D dataset (left) and OpenPose post-detection skeleton (right). . . . . . . . . . . . . . . 26

3.3 The input will be feed into a Batch Normalization (BatchNorm) layer and nine blocks of spatial-temporal graph convolution (ST-GCN) will be applied and gradually generate higher-level feature maps on the graph. It will then be classified by the standard Softmax classifier to the corresponding action category. . . . . . . . . 27 


\section{List of Tables}

3.1 Performance of three different kernel on NTU RGB+D dataset. Bold font indicates the best result . . . . . . . . . . . . . . . . . . . . . . . . 30

3.2 Performance of the application of the ST-GCN with three different graph connection approaches in our 2D skeleton-based ADTI dataset. 


\section{List of Abbreviations}

ASD Autism Spectrum Disorder

GCN Graph Convolutional Network

ADOS Autism Diagnostic Observation Schedule

ADOS-2 Autism Diagnostic Observation Schedule-2

LSTM Long Short-term Memory

CNN Convolutional Neural Networks

CSSs Calibrated Severity Scores

SBRI Stereotyped Behaviors Restricted Interests

IC Imagination/Creativity

RSI Reciprocal Social Interaction

ADI-R Autism Diagnostic Interview-Revised

DNN Deep Neural Network

XAI Explainable Artificial Intelligence

FSIQ Full-scale IQ

FC Fully Connected

BatchNorm Batch Normalization

ReLU Rectified Linear Unit 
ROC Receiver Operating Characteristic

HED-SC Holistically-Nested Edge Detector

SCQ Social Communication Questionnaire

AQ Autism Quotient

Caltech California Institute of Technology

SGD Stochastic Gradient Descent

LRP Layer-wise Relevance Propagation

AUC Area Under the Curve

RNN Recurrent neural network

GC Graph Convolution

TC Temporal Convolution

CV Cross-View

CS Cross-Subject

ADTI Autism Demonstration Task Interview 


\section{Chapter 1}

\section{Introduction}

How to improve the efficiency and effectiveness of diagnosing ASD has been explored in the last decade. The traditional approaches usually based on face to face interview. Recently, thanks to the advance in more powerful computing resources, deep learning techniques are rapidly developing. Convolutional Neural Networks $(\overline{\mathrm{CNN}})$ play more and more important role to solve most of the diagnosing ASD tasks.

\subsection{Motivation}

\subsubsection{Analysis of ASD First-Person Perspective}

The ability to attend to what is important in the environment is one of the most fundamental cognitive functions in humans. However, people with ASD show profound impairments in attention, especially to social stimuli such as human faces and social scenes [2-4]. Prior studies have documented that individuals without ASD spend significantly more time than peers with ASD looking at the eyes when viewing human faces presented in movie clips [5] or photographs [6]. When comparing social versus nonsocial stimuli, people with ASD show reduced attention to human faces and to other social stimuli such as the human voice and hand gestures; however, they pay more attention to nonsocial objects [7,8], notably including gadgets, devices, vehicles, electronics, and other objects of idiosyncratic "special interest" [9].

Sec. 1.1.1 and 1.2.1 (C2020 International Society for Autism Research and Wiley Periodicals LLC. Reprinted, with permission, from M. Ruan, P.J. Webster, X. Li, and S. Wang, Deep Neural Network Reveals the World of Autism From a First-Person Perspective, Autism Research, 2020. The reference can be found in [1]. 


\subsubsection{Video-based ASD Behavior Recognition}

With the development of society, the estimated ASD prevalence is dramatically increasing. Basically, the traditional diagnosis of ASD aims at improving observation measures and interviewing questions to elicit specific ASD behaviors. ADOS-2 [10] is the gold-standard for diagnosing ASD It focuses on four key areas: communication, reciprocal social interaction, imagination/creativity, and stereotyped behaviors/restricted interests. However, most of the ASD diagnoses have to be done by specialists instead of the pediatricians who do not have significant extensive training in diagnosing ASD. Moreover, the lack of specialists makes the situation getting worse. Most of them have a backlog of patients from 6 to 12 months due to the long process of diagnosing. The combination of these two factors causes increasing delays in diagnosing ASD. According to the statistics, first signs of ASD often visible by one year of age, while the average age of diagnosis is over four years old in the U.S. Worse still, with the impacts of COVID-19, this situation is exacerbated by the increased demand for telehealth diagnosing ASD. Therefore, it is necessary to find out an effective and efficient method for diagnosing $\mathrm{ASD}$ based on video data.

However, existing studies regarding ASD have not explored the application of deep learning in the video-based diagnosing ASD task and human action recognition is one of the fine points of penetration. Recently, human action recognition has become an active research area, as it plays a significant role in video understanding. The crucial factor of this problem is how to extract the spatial-temporal information from the video input. In general, human action can be recognized from multiple modalities. The first one is the combination of convolutional and recurrent layers to the architecture [11, 12], such as Long Short-term Memory (LSTM), which can capture temporal sequence and long-range information, and then uses this information to encode the current state. LSTMs on the last convolutional layers can have a large receptive field but may not be able to capture fine low-level motion which is critical in many cases. Different from the LSTM mechanism, the two stream framework [13-15] extracted spatial and temporal features. They respectively fed a single RGB frame and a stack of pre-computed optical flow frames into two CNNs and then fused the prediction to calculate the final output. Another modality to model human actions is applied the 3D filter to the CNNs [16,17]. They seem like the standard CNN, but with the spatial-temporal kernel which directly aggregated hierarchical representations of spatial-temporal data. Nevertheless, 
these methods remain some limitation when the videos have a noisy background with complex occlusion or illumination conditions, changing camera view angles.

To solve these issues, we applied the ST-GCN [18] to our task. GCN architecture, different from CNNs only can learn non-Euclidean structure data(i.e. image), can be applied to most of the topology graph dataset. For the above reasons, the GCN architecture has been effectively applied to a number of areas and become active last several years, especially in the topic of human action recognition when its effectiveness is demonstrated by ST-GCN. In this model, every input is defined as an external sequence of physical connection graphs where skeleton joints and bones are defined as graph nodes and edges respectively. With this skeleton-based input, the model is better robust against occlusion, illumination, and viewpoint change.

However, the GCN aggregates information based on an unchanged graph, designed according to the physical connection, through all the layers. It means the kernel is static and only describes physical edges between neighboring nodes, thus, cannot capture dynamic spatial correlations properly. For example, when we are clapping or hugging, in the graph, two hands should have a strong dependency but there is no connection in the physical-based graph. Furthermore, with the data going deeper layers, the feature has a larger receptive field but the static kernel cannot match this change.

\subsection{Problem Statement}

In this dissertation, we mainly focus on two points of breakthrough regarding diagnosing ASD problems: 1) Analysis of ASD First-Person Perspective ;2) video-based ASD behavior recognition.

\subsubsection{Analysis of ASD First-Person Perspective}

In this study, we used deep learning to classify photos taken in three different categories (people, indoors, and outdoors) as either having been taken by individuals with ASD or by peers without ASD. Our classifier effectively discriminated photos from all three categories but was particularly successful at classifying photos of people with $>80 \%$ accuracy. Importantly, the visualization of our model revealed critical features that led to successful discrimination and showed that our 
model adopted a strategy similar to that of ASD experts. Furthermore, for the first time, we showed that photos taken by individuals with ASD contained less salient objects, especially in the central visual field. Notably, our model outperformed the classification of these photos by ASD experts. Together, we demonstrate an effective and novel method that is capable of discerning photos taken by individuals with ASD and revealing aberrant visual attention in ASD from a unique first-person perspective. Our method may in turn provide an objective measure for evaluations of individuals with ASD.

\subsubsection{Video-based ASD Behavior Recognition}

In this work, we propose to address the above issue (Sec. 1.1.2) by applying the skeleton-base ST-GCN [18] action recognition model to our ASD behavior recognition task. GCN architecture, different from $\mathrm{CNNs}$ only can learn non-Euclidean structure data(i.e. image), can be applied to most of the topology graph dataset. For the above reasons, the GCN architecture has been effectively applied to a number of areas and become active last several years, especially in the topic of human action recognition when its effectiveness is demonstrated by ST-GCN. In our study, every input is defined as an external sequence of physical connection graphs where skeleton joints and bones are defined as graph nodes and edges respectively. With this skeleton-base input, our model is better robust against occlusion, illumination, and viewpoint change, and especially the background bias in our ASD interview data, where the participants with ASD and controls are interviewed respectively in two rooms. Moreover, the model has a fine expressive power in extracting features in both temporal and spatial domains. To further improve its aggregation capacity, we introduced the attention mechanism which allows the network to dynamically learn an adaptive graph during the training process and concentrate its computational resources on the most useful features and enhance the discriminative learning ability. Our preliminary model is tested on two datasets. The first one is a large-scale action recognition dataset called NTU-RGBD. And the second one is our ASD interview videos.

\subsection{Contributions}

1. Analysis of ASD First-Person Perspective 
- We propose the application of the VGG16 to our analysis of ASD first-person perspective task;

- We propose the visualization of our model revealed critical features that led to successful discrimination and showed that our model adopted a strategy similar to that of ASD experts;

- For the first time, we showed that photos taken by individuals with ASD contained less salient objects, especially in the central visual field;

- We tested our model and demonstrate that our model can outperform autism experts in our dataset.

2. Video-based ASD Behavior Recognition

- We propose the application of GCN in our $\mathrm{ASD}$ behavior recognition task;

- We propose to address the issue of the fixed input graph for action recognition and introduce the attention mechanism to the GCN, which can learn an adaptive graph for the model;

- We tested our model on a large-scale action recognition dataset called NTU-RGBD, and our ASD interview videos. Experimental results have shown our model can significantly improve the performance.

\subsection{Dissertation Structure}

The rest of this dissertation is organized as follows:

- Chapter 2 reviews the ASD stimuli approaches and introduces the application of VGG16 to our analysis of ASD first-person perspective task. Moreover, it introduces a method how we evaluate and explain our model.

- Chapter 3 reviews the main action recognition modalities and introduces to application of STGCN to our ASD behavior recognition task. Furthermore, it introduces an adaptive graph to improve the performance.

- Chapter 4 presents the conclusions of this dissertation and plans the future works. 


\section{Chapter 2}

\section{Analysis of ASD First-Person Perspective}

\subsection{Related Work}

In order to better understand the atypical social behavior in ASD, there is an increasing trend to employ more natural and ecologically valid stimuli (e.g., complex scenes taken with a natural background) [3, 19, 25] and to test participants in a more natural setting as opposed to a restricted clinical setting in which core ASD behaviors may not be seen. Tasks presenting faces in a naturalistic setting demonstrate that people with ASD have reduced attention to faces and specifically to the eye region [5, 26, 28, 28, 29]. In particular, recent studies have directly tested people with ASD during natural interactions with lab personnel [30-32]. For example, we asked ASD participants and controls to take photos in natural social settings and showed that while those with ASD take more photos of people than controls, those photos are more often not front-facing and/or are taken from odd perspectives indicating a lack of social engagement [32].

Recent studies have been employing machine learning techniques to quantitatively characterize atypical behavior in ASD. In addition to its benefits of improving and streamlining ASD diagnosing [33-35], machine learning can reveal critical features of atypical behavior in ASD from various domains of data, including eye movement [3, 36], scoring of Autism Diagnostic InterviewRevised (ADI-R [35], scoring of Autism Diagnostic Observation Schedule (ADOS] [33], and home videos [34]. Therefore, machine learning can provide an effective and crucial way for us to

(C2020 International Society for Autism Research and Wiley Periodicals LLC. Reprinted, with permission, from M. Ruan, P.J. Webster, X. Li, and S. Wang, Deep Neural Network Reveals the World of Autism From a First-Person Perspective, Autism Research, 2020. The reference can be found in [1]. 
identify and understand the factors that contribute to atypical behavior in ASD.

The current study explored the feasibility of using a Deep Neural Network (DNN) to discern between photos taken by participants with ASD versus those taken by controls. These photos provide a unique first-person perspective of what is visually salient to the photographer and reflect their social interactions with their environment not seen in other studies in which those with ASD are asked to view stock photos. Therefore, by classifying these photos, we were able to reveal aspects of aberrant visual attention in individuals with ASD. Indeed, our deep neural network could effectively classify whether a photo was taken by an individual with ASD or by a peer without ASD, Surprisingly, our machine-based approach consistently outperformed human-based classification ratings conducted by ASD experts. Notably, our Explainable Artificial Intelligence (XAI) technique revealed the critical features that support the classification. Together, we showed that photos taken from a first-person perspective by those with ASD can aid in understanding their unique visual perspective of the world and that deep neural networks may provide an efficient and objective method to aid in the analysis of visual attention deficits in ASD.

\subsection{Dataset and Materials}

\subsubsection{Participants}

All participants were from our previous report [32]. Briefly, 16 high-functioning participants with ASD (12 male) and 21 controls (18 male) were recruited (Supplementary Table S1 A). All ASD participants met DSM-5/ICD-10 diagnostic criteria for ASD and all met the cutoff scores for ASD on the ADOS-2 revised scoring system for Module 4 [37], and the ADI-R [38, 39] or Social Communication Questionnaire (SCQ) [40]. The ASD group had a Full-scale IQ (FSIQ) of $111.6 \pm$ 12.2 (mean $\pm \mathrm{SD}$, from the Wechsler Abbreviated Scale of Intelligence-II), the mean age of $29.7 \pm$ 11.2 years, and a mean Autism Quotient $\mathrm{AQ}$ of $29.7 \pm 8.07$. Controls had a comparable FSIQ of $111.0 \pm 9.90$ (t-test, $\mathrm{P}=0.92$, although IQ was only available on a subset) and a comparable mean age of $33.0 \pm 9.31$ years ( $\mathrm{t}$-test, $\mathrm{P}=0.33$ ). The groups were also matched for gender, race, and education (Supplementary Table S1 A). Participants provided written informed consent according to protocols approved by the institutional review board of the California Institute of Technology 
(Caltech), and all methods were carried out in accordance with the approved guidelines.

\subsubsection{Task}

Participants were provided with a camera and instructed to take photos of anything they wanted, such as objects, rooms, scenery, or people, and they could take as many photos as they wished. There were three blocked conditions (in counterbalanced order between participants):

\section{People Block}

Photographing for this block took place in the rooms and hallway of a Caltech laboratory. Participants were instructed to primarily take photos of two lab members, who were fully aware of the experiment and thus were prepared to pose or be expressive. Some participants with ASD were also instructed to take self-portraits. Participants were free to set up space however they liked (e.g., they could move around the room or interact with the objects in the room) and they could also ask the two lab members to move or pose to their instruction.

\section{Indoor Block}

Photographing took place in the same indoor environment and participants were instructed to walk around the lab and feel free to enter lab spaces to photograph objects.

\section{Outdoor Block}

Photographing took place on the Caltech campus outside of the building. Participants were instructed to walk anywhere on campus if they wished and take photos of any objects or people of their own choosing.

During each condition, participants were asked to take at least ten photos. Five participants with ASD completed two sessions of the experiment and we have pooled photos from both sessions for each participant for analysis. 


\subsubsection{Rating by ASD Experts}

ASD experts skilled in ASD evaluations using the ADOS rated all photos independently on a 9 point scale $(1=$ the photo was definitely taken by a person with ASD to $9=$ the photo was definitely taken by a person without ASD; see [32] for detailed instructions for photo ratings). Photos, especially those taken by participants with ASD of themselves, were excluded from rating if an ASD expert could recognize the identity of the participant so that all raters remained blinded to which group (ASD or control) the participant belonged. All photos were shown in randomized order within each of the three photo task conditions (People, Indoors, Outdoors). Notably, these professional raters were highly consistent in their ratings [32].

\subsubsection{First-Person Perspective Dataset}

There was a total of 1672 photos in our dataset (Supplementary Table S1 A). The People Block contained 490 photos from participants with ASD and 217 photos from controls. The Indoor Block contained 265 photos from participants with ASD and 229 photos from controls. The Outdoor Block contained 229 photos from participants with ASD and 242 photos from controls. Photos from all participants were pooled for training and testing. To augment the dataset, before training, the input images were randomly cropped to a random size and then rescaled to $224 \times$ 224 RGB images, meanwhile, the images had a 0.5 probability to be horizontally flipped. In each training/testing run (separately for each task condition), the dataset was randomly split into three parts: $60 \%$ served as the training set, $20 \%$ served as the validation set, and $20 \%$ served as the test set. We repeated the procedure 10 times with different random splits of training and testing data. Additional different splits of training and testing data were also tested and we derived qualitatively the same performance (see Results).

\subsection{Methods}

\subsubsection{Classification}

We have adopted a strategy of fine-tuning a pre-trained VGG16 convolutional neural network (CNN) on ImageNet to discriminate photos taken by participants with ASD from those taken by 
A

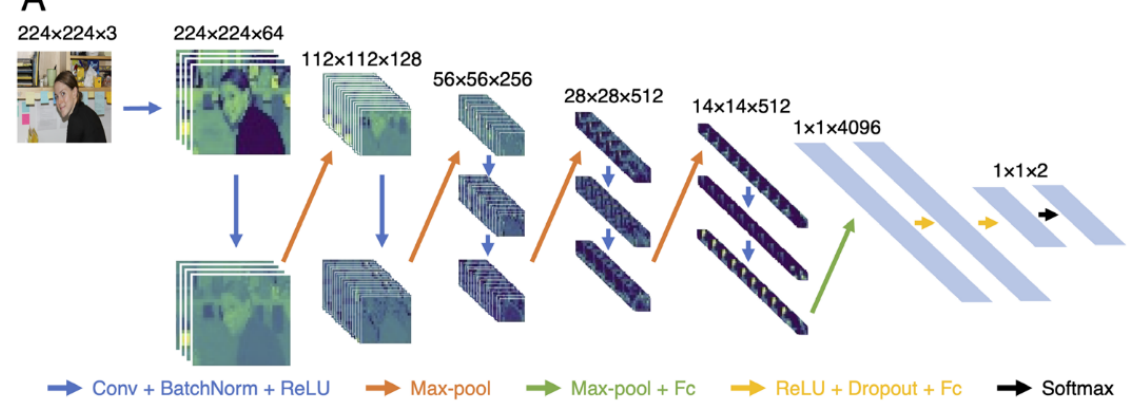

B

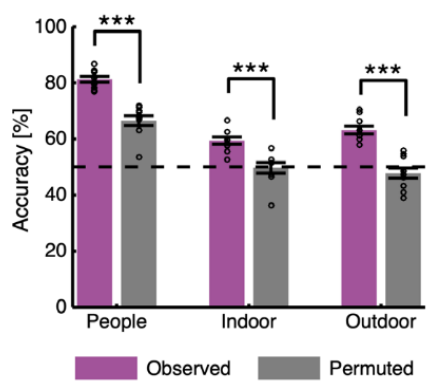

Figure 2.1: Model architecture and performance. (A) Model architecture. The input was a fixedsize $224 \times 224$ RGB image. The image was passed through a stack of convolutional layers, where the filters were used with a very small receptive field of $3 \times 3$. The convolution stride was fixed to 1 pixel; the spatial padding of convolutional layer input was such that the spatial resolution was preserved after convolution (i.e., the padding was 1-pixel for $3 \times 3$ convolutional layers). Spatial pooling was carried out by five max-pooling layers, which followed some of the convolutional layers. Max-pooling was performed over a $2 \times 2$ pixel window, with stride 2 . Three fully connected (FC) layers followed a stack of convolutional layers: the first two had 4096 channels each, the third performed a two-way ASD classification and thus contained two channels. The final layer was the Softmax layer. All hidden layers were equipped with the rectification (ReLU) nonlinearity. (B) Model prediction accuracy. Our model could differentiate photos taken by those with ASD from those taken by controls in all conditions. Error bars denote \pm SEM across runs and circles show individual values. Asterisks indicate a significant difference in prediction accuracy between observed (magenta) versus permuted (gray) runs using unpaired t-test: $* * *: \mathrm{P}<0.001$. 
controls (Fig. 2.1A). This CNN is capable of recognizing a large class of objects [41], including faces, indoor objects, and outdoor objects, and is thus suitable for our task to analyze first-person perspective. The CNN consisted of a feature extraction section (13 convolutional layers) and a classification section (three Fully Connected (FC) layers). The feature extraction section was consistent with the typical architecture of a CNN. We applied a $3 \times 3$ filter with 1-pixel padding and 1-pixel stride to each convolutional layer, which followed by a BatchNorm and Rectified Linear Unit ( $\mathrm{ReLU})$ operation. Some of the convolutional layers were followed by five $2 \times 2$ max- pool operations with a stride of 2. There were three FC layers in each classification section: the first two had 4096 channels each, and the third performed a two-way ASD classification and thus contained two channels. Each FC layer was followed by a ReLU and 50\% dropout to avoid overfitting. A nonlinear Softmax operation was applied to the final output of the VGG16 network to make the binary classification prediction. It is worth noting that the classification was performed and fine-tuned for each task condition separately.

\subsubsection{Receiver Operating Characteristic Curves}

We used $\mathrm{ROC}$ curves to evaluate and compare classification performance. We constructed two kinds of ROC curves, respectively calculated from professional rating scores and our model prediction output, as a comparison. For the rating scores, three independent ASD experts familiar with the clinical presentation of ASD and research reliable on the ADOS-2 were asked to evaluate and score every photo from our dataset. The score represents how confident they are that the photo was taken by a participant with ASD or a control (scores from 1 to $9 ; 1=$ confident the photo was taken by a participant with ASD, $9=$ confident the photo was taken by a control participant). Thus, curves of human performance were constructed based on the experts' average scores for each photo.

For our model, following the last layer, the Softmax operation output the probability distribution of the predictions as positive (a participant with ASD took the photo) or negative (a control participant took the photo). The prediction probabilities were used to construct the ROC curve. To reduce bias in the dataset, we tested our model 10 times with ten different splits of training and testing data; but every shuffle of the training, validation, and test datasets was based on the 
same split ratio. The AUC of the $\mathrm{ROC}$ was calculated by integrating the area under the $\mathrm{ROC}$ curve (trapezoid rule). Note that since $\mathrm{ROC}$ is a probability curve, $\mathrm{AUC}$ indicates the degree or measure of separability (i.e., tells how well the model is capable of distinguishing between classes such as ASD versus control).

\subsubsection{Saliency Analysis}

To detect salient objects in the photos, we applied the most recent saliency detection algorithm to extract the saliency map from an input photo [42]. This algorithm applies short connections to the skip-layer structures within a Holistically-Nested Edge Detector (HED-SC). By taking full advantage of multilevel and multiscale features extracted from fully convolutional neural networks, HED-SC can offer fine-granularity representations at each layer leading to state-of-the-art saliency detection performance. We calculated the average saliency values for the two regions. The central region consisted of a rectangle located at the image center and sized by $1 / 3$ width $\times 1 / 3$ height of the image; and the peripheral region consisted of the rest of the image.

\subsection{Experimental Results}

In this section, we demonstrate the network setting, training details, explanation, and experimental results of model performance and saliency analysis. Finally, compare our model with human performance.

\subsubsection{Implementation details}

\section{Training the DNN}

Our VGG16 network ran on the deep learning framework of PyTorch [43, 44]. To improve model performance with our small dataset, we have applied Transfer Learning to our model. For the feature extraction part, we loaded the pre-trained weights on ImageNet and froze the convolutional layers to prevent their weights from updating during training. With a better feature extraction, our dataset was mainly used to train the FC layers to improve its ability of classification. The training was performed by the Stochastic Gradient Descent (SGD) optimizer with a base 
learning rate of $10^{-3}$.

\section{Permutation test}

To further confirm the results, statistical significance was estimated by permutation test. There were ten runs, and in each run, photo labels were randomly shuffled and the training/testing procedure was repeated. P-values were calculated by comparing the observed accuracy to that from the permutation test.

\subsubsection{Model Performance}

DNN performance was above chance for all conditions, including the People Block (permuted: $66.5 \% \pm 5.49 \%$; unpaired two-tailed t-test between observed versus per- muted performance: $\mathrm{t}(18)$ $=7.27, \mathrm{P}=9.33 \times 10^{-7}$ ), the Indoor Block (permuted: $49.7 \% \pm 5.93 \% ; \mathrm{t}(18)=4.30, \mathrm{P}=4.32 \times$ $10^{-4}$ ), as well as the Outdoor Block (permuted: $47.8 \% \pm 5.73 \% ; \mathrm{t}(18)=6.79, \mathrm{P}=2.34 \times 10^{-6}$; Fig. 2.1B), demonstrating that the VGG model could be applied to successfully discriminate all categories of photos as having been taken by people with ASD, but photos of people were the most discriminative. Notably, our model performance still held when we excluded all self-portraits from the People Block $\left(80.8 \% \pm 3.13 \% ; \mathrm{P}=1.13 \times 10^{-6}\right)$, suggesting that our classification was not simply driven by self-portraits from participants with ASD (note that only participants with ASD took self-portraits). Our results were also consistent with our prior published report from ASD experts experienced in ADOS administration demonstrating that photos from the People Block are most discriminative between participants with ASD and controls. It is worth noting that ASD experts did not successfully discriminate photos from the Outdoor Block as having been taken by those with versus without ASD [32].

We conducted the following control analyses to confirm our model performance. (1) We employed a tenfold cross-validation that derived $83.7 \% \pm 3.87 \%$ accuracy for the People Block, $64.6 \% \pm 6.60 \%$ accuracy for the Indoor Block, and 59.3\% $\pm 5.92 \%$ accuracy for the Outdoor Block. (2) We further tested model bias by randomly assigning a label to each photo (i.e., each photo had a 50\% probability to be labeled as ASD or control). We derived a chance performance (i.e., 50\% accuracy) for all three conditions: we derived $47.3 \% \pm 14.3 \%$ accuracy for the Peo- 
ple Block (paired t-test against 50\%: $\mathrm{P}=0.57$ ), 50.8\% $\pm 6.58 \%$ accuracy for the Indoor Block $(\mathrm{P}=0.70)$, and $47.9 \% \pm 6.57 \%$ accuracy for the Outdoor Block $(\mathrm{P}=0.33)$, suggesting that our model was not biased toward reporting one category of photos. (3) Consistent with (2), we derived similar results (People Block: accuracy $=77.0 \% \pm 5.58 \%, \underline{\mathrm{AUC}}=0.85 \pm 0.052$; Indoor Block: accuracy $=63.3 \% \pm 3.50 \%, \widehat{\mathrm{AUC}}=0.68 \pm 0.052$; Outdoor Block: accuracy $=64.2 \% \pm 5.24 \%$, $\mathrm{AUC}=0.71 \pm 0.048$ ) when we used an equal number of photos from participants with ASD and controls, suggesting that the results could not simply be attributed to more photos having been taken by participants with ASD (4) Although blurred photos are a major characteristic indicating ASD [32] (also see below in Fig. 2.2), when we excluded all blurred photos from the analysis, we still derived similar results (People Block: accuracy $=81.3 \% \pm 4.50 \%, \widehat{A U C}=0.91 \pm 0.030$; Indoor Block: accuracy $=64.7 \% \pm .73 \%, \widehat{\mathrm{AUC}}=0.69 \pm 0.060$; Outdoor Block: accuracy $=61.0 \%$ $\pm 5.77 \%, \widehat{\mathrm{AUC}}=0.68 \pm 0.062$ ). (5) We conducted a leave-one-participant-out analysis by training the classifier with photos from all but one participant and testing on the remaining participant. We found an above-chance performance (People Block: accuracy $=61.7 \% \pm 27.9 \%[$ mean $\pm \mathrm{SD}$ across participants/validations], $\mathrm{AUC}=0.72$; Indoor Block: accuracy $=55.8 \% \pm 25.0 \%$, $\mathrm{AUC}$ $=0.59$; Outdoor Block: accuracy $=59.7 \% \pm 23.2 \%, \underline{\mathrm{AUC}}=0.60)$, suggesting that photos from different participants within a group shared similar features and our model could generalize to predict whether a new photo was taken by a control participant or a participant in the ASD group. The People Block still showed the best performance, likely because the way that participants from each group composed the photos was more consistent. Note that AUC could only be assessed if we pooled photos from different participants because there was only one label for each participant; and we derived an accuracy of $67.0 \%$ for the People Block, an accuracy of $56.8 \%$ for the Indoor Block, and an accuracy of $58.6 \%$ for the Outdoor Block if we pooled all photos to assess prediction performance. We next explored the factors that led to correct classification.

\subsubsection{Model Explanation Through Layer-Wise Relevance Propagation}

To provide an explanation of our model's output in the domain of its input, we applied Layerwise Relevance Propagation ( $(\overline{\mathrm{LRP}})$ to our trained classifier. LRP can use the network weights created by the forward-pass to propagate the output back through the network up until the original 


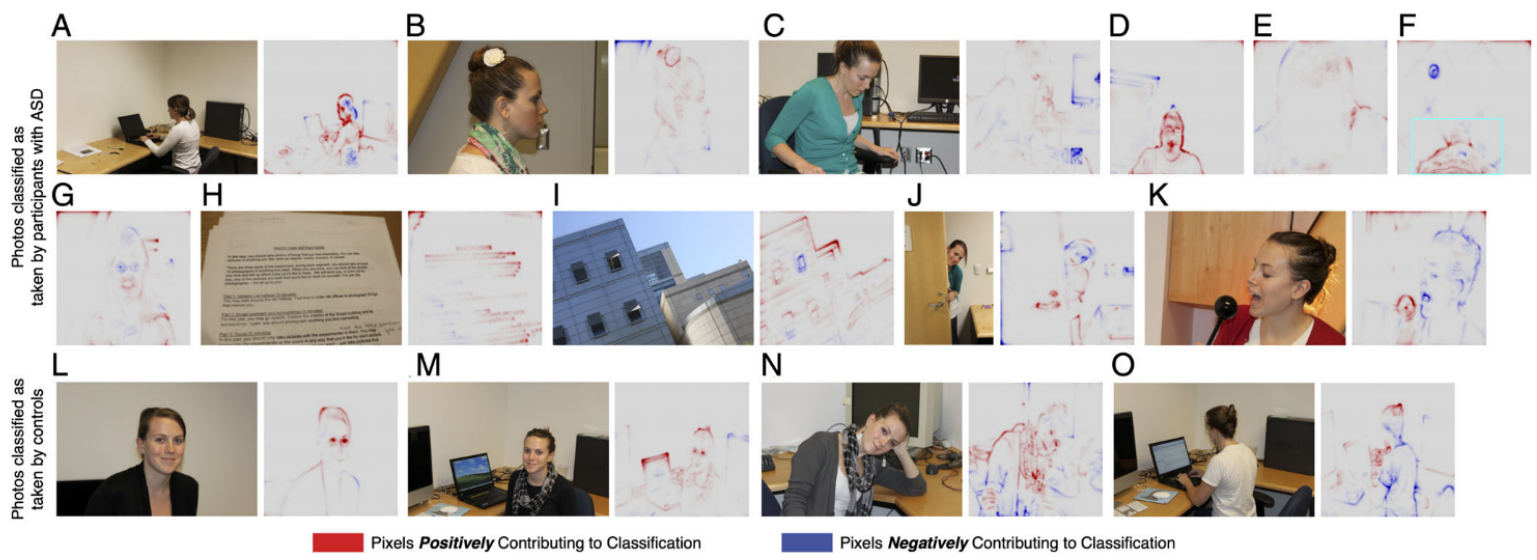

Figure 2.2: Layer-wise Relevance Propagation (LRP) explanation. (A-K) Photos that were classified as having been taken by participants with ASD, (L-O) Photos that were classified as having been taken by controls. Red pixels positively contributed to the classification whereas blue pixels negatively contributed to the classification. $(\mathrm{J}, \mathrm{K})$ Photos that were incorrectly classified as having been taken by participants with ASD. $(\mathrm{N}, \mathrm{O})$ Photos that were incorrectly classified as having been taken by controls. The cyan box in (F) illustrates where the subject of the photo was located.

input image. The explanation given by LRP is a heatmap of which pixels in the original image contribute to the final output (Fig. 2.2; red pixels positively contributed to the classification whereas blue pixels negatively contributed to the classification). In the People Block, we found that classification of a photo as having been taken by participants with ASD was driven by the following factors: (1) photos had a view of the subject's back (Fig. 2.2A) or side (Fig. 2.2B); (2) subjects in the photos did not pose or look at the camera (Fig. 2.2A-C); (3) subjects in the photos were not expressive (Fig. 2.2B,C); (4) photos had an odd visual perspective (Fig. 2.2D-F; the cyan box in Fig. 2.2F denotes where the subject was located); and (5) photos were blurred (Fig. 2.2 G; also note that the eyes in this photo looked at the camera and negatively contributed to classifying this photo as taken by participants with ASD [as shown in blue]). In contrast, photos with rich facial expressions and a regular angle of view (Fig. 2.2 L,M) would lead to our classifier identifying them as having been taken by controls. Similarly, in the Indoor Block and Outdoor Block, photos that were blurred (Fig. 2.2 $\mathrm{H}$ ) or slanted (Fig. 2.2 $)$ were classified as having been taken by participants with ASD. These features derived from machine learning were consistent with the intuition of ASD experts from our prior study [32], suggesting that both approaches (human ratings and DNN) adopted a similar strategy in discriminating the photos.

Notably, in the photos that were mistakenly classified as being taken by participants with ASD 

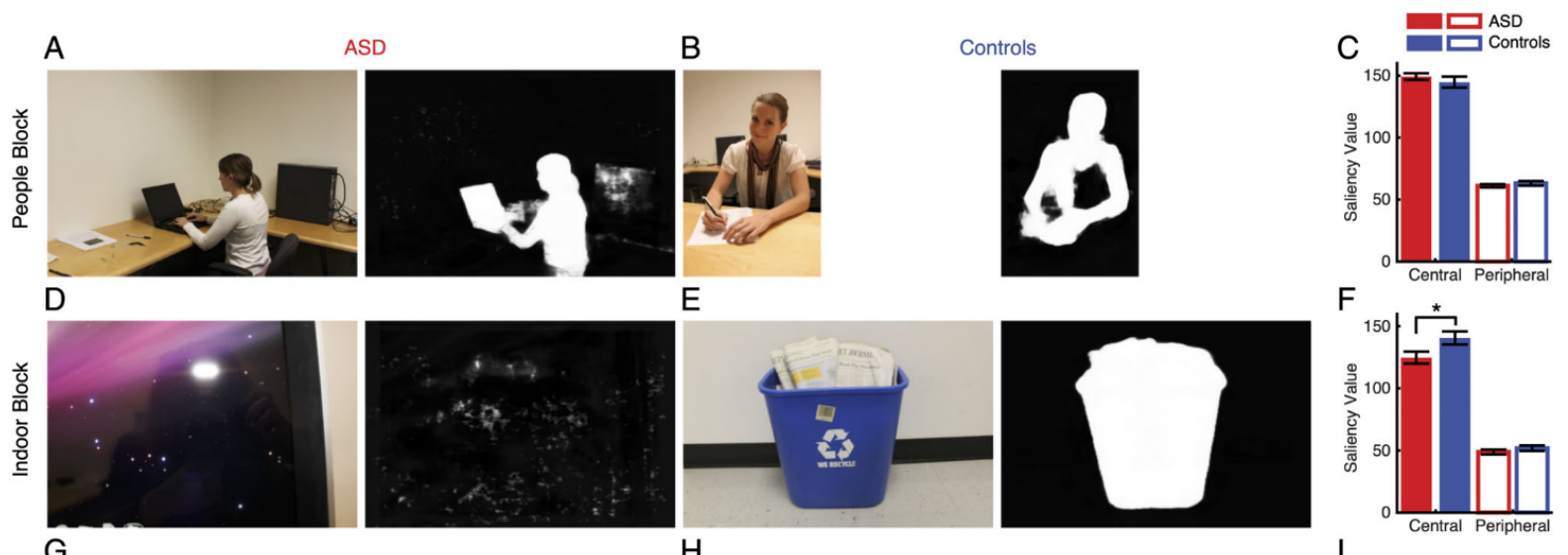

E

$\mathrm{F}$
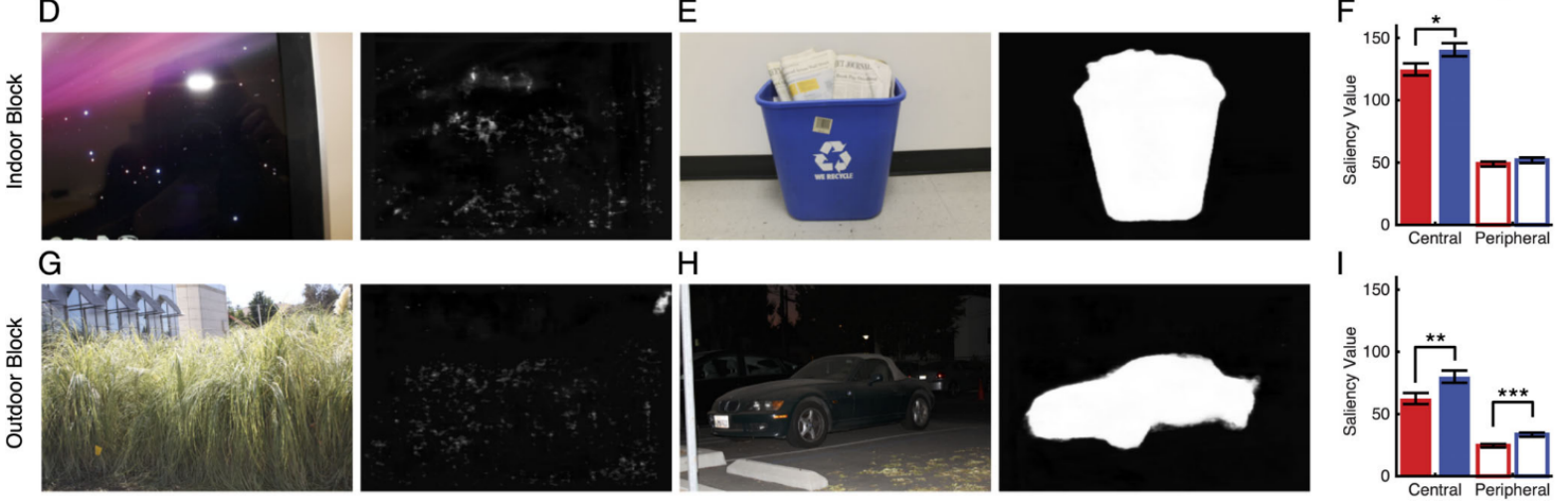

$\mathrm{H}$

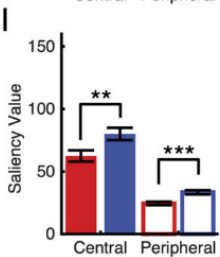

Figure 2.3: Saliency analysis. (A-C) People Block. (D-F) Indoor Block. (G-I) Outdoor Block. (A, B, D, E, G, H) Example photos (left) with detected saliency maps (right). (A, D, G) Photos were taken by participants with ASD (B, E, H) Photos taken by controls. (C, F, I) Average saliency value. Error bars denote \pm SEM across photos. Solid bars denote the central region and open bars denote the peripheral region. Asterisks indicate significant difference between ASD and controls using two-tailed unpaired t-test: *: $\mathrm{P}<0.05, * *: \mathrm{P}<0.01$, and $* * *$ : $\mathrm{P}<0.001$. Red: ASD Blue: controls.

(Fig. 2.2J,K), front view of the face (Fig. 2.2J) and facial expressions (Fig. 2.2K) still contributed to the classification of the photos as having been taken by controls (i.e., negatively contributed to classifying the photos as from participants with $\mathrm{ASD}$ ). On the other hand, in the photos that were mistakenly classified as being taken by controls (Fig. $2.2 \mathrm{~N}, \mathrm{O}$ ), the front view of the face and rich facial expressions (Fig. $2.2 \mathrm{~N}$ ) led to such classification whereas the view of the subject's back (Fig. 2.2 $\mathrm{O}$ ) still contributed to the classification of the photos as having been taken by participants with ASD (i.e., negatively contributed to classifying the photos as from controls). Therefore, these results suggest that our classifier adopted a consistent strategy in classifying the photos and the incorrectly classified photos might be driven by other factors.

\subsubsection{Saliency Analysis of Photos}

Since participants with ASD demonstrate atypical visual saliency [3], we employed a saliency model to detect salient objects in the photos (see Methods). We found that in the People Block 
(Fig. 2.3A-C), photos taken by participants with ASD had similar saliency values compared to those taken by controls for both the central region (ASD: $149 \pm 59.8$ [mean \pm SD], controls: 145 \pm 65.2 ; two-tailed unpaired t-test: $\mathrm{t}(705)=0.90, \mathrm{P}=0.37$, effect size in Hedges' $\mathrm{g}$ (standardized mean difference): $\mathrm{g}=0.073$, permutation $\mathrm{P}=0.37$ ) and the peripheral region (ASD: $61.1 \pm 29.4$, controls: $63.1 \pm 27.6 ; \mathrm{t}(705)=0.82, \mathrm{P}=0.41, \mathrm{~g}=0.067$, permutation $\mathrm{P}=0.37)$. In the Indoor Block (Fig. 2.3D-F), photos taken by participants with ASD were less salient in the central region (ASD: $125 \pm 79.2$, controls: $141 \pm 79.5 ; \mathrm{t}(492)=2.20, \mathrm{P}=0.028, \mathrm{~g}=0.20$, permutation $\mathrm{P}=0.036$ ) but not in the peripheral region (ASD: $48.9 \pm 31.7$, controls: $51.9 \pm 33.1 ; \mathrm{t}(492)=1.00, \mathrm{P}=0.32$, $\mathrm{g}=0.091$, permutation $\mathrm{P}=0.32)$. Notably, in the Outdoor Block (Fig. $2.3 \mathrm{G}-\mathrm{I})$, photos taken by participants with ASD were less salient in both the central region (ASD: $62.5 \pm 68.2$, controls: $80.0 \pm 76.5 ; \mathrm{t}(469)=2.62, \mathrm{P}=0.009, \mathrm{~g}=0.24$, permutation $\mathrm{P}=0.008)$ and the peripheral region (ASD: $24.5 \pm 20.8$, controls: $33.4 \pm 26.5 ; \mathrm{t}(469)=4.05, \mathrm{P}=5.87 \times 10^{-5}, \mathrm{~g}=0.37$, per- mutation $\mathrm{P}$ $<0.001)$. As expected, the central region of the photos was more salient than the peripheral region (all Ps $<10^{-14}$ ). Together, our results suggest that photos taken by participants with ASD contained less salient objects compared to photos taken by controls, especially in the central region of the visual field. Notably, this new finding was not revealed in our previous human evaluations [32].

\subsubsection{Comparison With Human Performance}

Lastly, we compared our DNN model with human ASD experts who scored each photo based on the degree to which they thought it had been taken by a participant with ASD or by a control participant (Fig. 2.4). For the People Block, the AUC value for the model's performance was $0.89 \pm 0.024$ (mean $\pm \mathrm{SD}$ across runs) and human performance was $0.66 \pm 0.046$ (Fig. 2.4A,B; paired t-test; $\mathrm{t}(9)=16.8, \mathrm{P}=4.15 \times 10^{-8}, \mathrm{~g}=5.85$, permutation $\mathrm{P}<0.001$; note that in each run, the test data were the same for the current machine learning analysis and for ratings by ASD experts). For the Indoor Block, the AUC value for model performance was $0.66 \pm 0.059$ and human performance was $0.66 \pm 0.055$ (Fig. 2.4 $\mathrm{C}, \mathrm{D} ; \mathrm{t}(9)=0, \mathrm{P}=1.0, \mathrm{~g}=1.87 \times 10^{-15}$, permutation $\mathrm{P}=$ 0.99). For the Outdoor Block, the AUC value for model performance was $0.70 \pm 0.051$ and human performance was $0.49 \pm 0.066$ (Fig. 2.4 $\mathrm{E}, \mathrm{F} ; \mathrm{t}(9)=7.12, \mathrm{P}=5.57 \times 10^{-5}, \mathrm{~g}=3.34$, permutation $\mathrm{P}$ $<0.001)$. Together, this result suggests that our classifier could generally outperform professional 

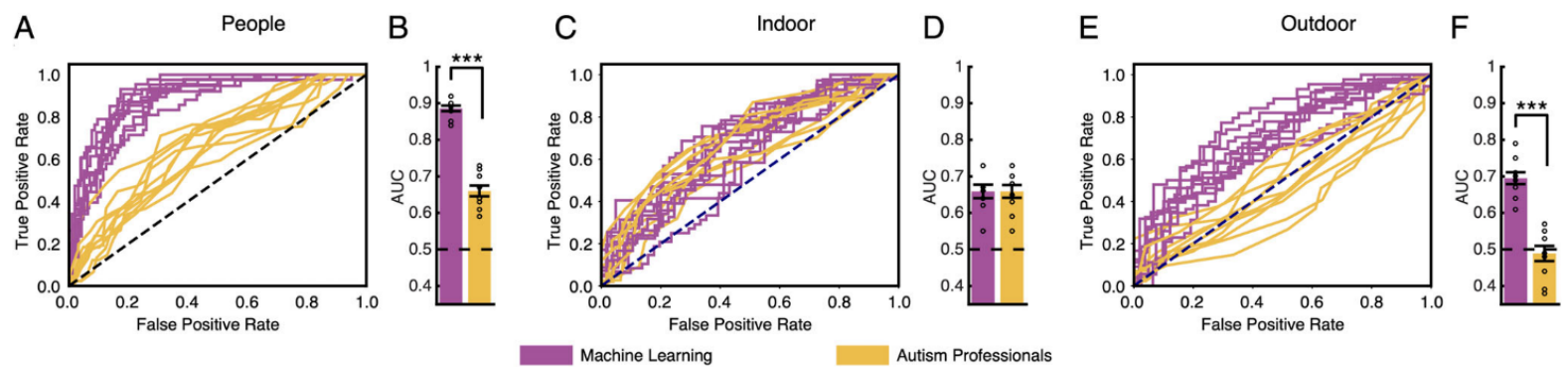

Figure 2.4: Our classifier model outperformed human ASD experts. (A, B) People Block. (C, D) Indoor Block. (E, F) Outdoor Block. (A, C, E) ROC curves. (B, D, F) The area under the ROC curve (AUC values). Error bars denote \pm SEM across runs and circles show individual values. Asterisks indicate a significant difference in performance between machine learning (purple) and ASD professionals (yellow) using paired t-test: ***: $\mathrm{P}<0.001$.

ASD experts and might be used as an effective method to better understand visual attention in ASD and to possibly diagnose individuals with $\mathrm{ASD}$.

\subsection{Discussion}

In this study, we developed a machine-learning algorithm that can effectively discriminate photos taken by participants with ASD from photos taken by controls. Despite the relatively small size of our training data, our VGG- based algorithm has shown consistent discriminating performance. Importantly, our analysis revealed critical features that led to such successful discrimination and showed that photos taken by participants with ASD con- tained less salient objects, especially in the central visual field. Notably, our machine learning-based ASD classification even outperformed classification by human ASD experts. Together, our findings can provide deeper insight into aberrant visual attention in ASD from a unique first-person perspective, which may in turn serve as a useful objective diagnostic tool for ASD

Our findings also suggest that photos taken of people were the most relevant to discern between photos taken by ASD participants from those taken by controls, consistent with general social deficits in ASD [45]. Given that participants needed to communicate with the person being photographed when the photos in the People Block were taken, the photos from the People Block not only showed how participants with ASD perceived other people but also reflected the degree to which they communicated with others. Therefore, our results also reflect deficits in 
social communication and interaction typical of ASD. It is worth noting that the experimenters being photographed had abundant experience working with participants with ASD, so they were very comfortable around individuals with ASD. Although the experimenters were not blinded to which participants had ASD, they were instructed to respond to participants with ASD and controls similarly.

It is also interesting to note that participants with ASD tended to take more photos of people than did controls. This observation has not been exploited by the current machine learning algorithm but suggests that participants with ASD may prefer to use a camera as a surrogate interface for interacting with other people. Furthermore, ASD is highly heterogeneous at the biological and behavioral levels [46]; and all participants involved in this study were high-functioning since our photographing task required cognitive abilities to use the camera as well as basic social communication skills to interact with others when they took photos of the other people. Therefore, our results may not apply to all individuals across the autism spectrum. However, machine learning has immense potential to enhance diagnostic and intervention research in the behavioral sciences and may be especially useful considering the heterogeneous nature of ASD [47].

The computational framework developed in this study can be readily extended to future studies that investigate other complex human social behavior and/or other neurological conditions. There have been several studies that have used photos to reveal predictive markers of psychiatric and/or neurological disorders. For example, Instagram photos have been found to reveal predictive markers of depression [48]. Along this line of research, deep learning has great potential to support the use of first-person perspective photos as predictive markers of visual attention deficits for other neurological disorders such as stroke and traumatic brain injury. Our present results further demonstrate that deep learning is more accurate at discerning whether a photo represented an ASD perspective and is more efficient than human-based photo classification which requires highly skilled ASD experts and is very time-intensive. Therefore, research investigating visual attention and diagnostic methods may benefit from the addition of first-person photos and DNNs to analyze those photos.

It is worth noting that there is a subtle difference when we compared the discriminating performance between ASD experts and machine learning. While machine learning using the actual photos as the training data, ASD experts did not have any training on this particular photo discrim- 
ination task nor did they receive any feedback about their performance. Rather, ASD experts had to use their knowledge or intuition to make their judgments. A future study will be needed to compare machine learning with human raters (even non-ASD experts) who are similarly trained on the photo discrimination task. Another possible extension of this study is to take first-person perspective videos. Despite some effort of machine learning-based ASD analysis using home videos [34], there is still no database of first-person perspective videos taken by individuals with ASD. We hypothesize that video clips taken by ASD patients may contain more useful and discriminative information than static photos (e.g., motion-related saliency information is often easier to characterize from a sequence of images rather than from a single image). We leave such an extension of this work a possibility in a future study.

More broadly, in line with our present results, there have been efforts to collect data from the first-person perspective of participants using head-mounted cameras or head-mounted eye trackers [49,50] . These egocentric-view data have revealed valuable information about how infants perceive faces over their first year of life [51], how social attention is coordinated between infants and parents [52], how people navigate in a cluttered environment [53], and how a brain lesion patient looks at faces of other people during conversations [54]. A clear future direction will be to apply head-mounted cameras or head-mounted eye trackers to record egocentric views from people with ASD during their real interactions with other people and the environment. These videos will provide the most direct data about how people with ASD perceive the world from their first-person perspective. Furthermore, continuous recordings from head-mounted cameras or head-mounted eye trackers can generate massive amounts of data (notably in comparison with static photos like those used in our present study). Therefore, deep learning, which has already shown promise to discriminate ASD from controls using eye movement data with natural scene images [36, 55], will make an important contribution to the analysis and interpretation of such egocentric-view data. 


\section{Chapter 3}

\section{Video-based ASD Behavior Recognition}

\subsection{Related Work}

Recently, human action recognition has become an active research area, as it plays a significant role in video understanding. The crucial factor of this problem is how to extract the spatialtemporal information from the video input. In general, human action can be recognized from multiple modalities, shown in Fig. 3.1. In this section, we review four main action recognition architectures: 1) CNN + LSTM; 2) Two Stream Network; 3) 3D CNN; 4) Skeleton-base GCN.

\subsubsection{CNN + LSTM}

To extract the temporal information, the first modality used recurrent layers to the CNN [11,12], such as LSTMs, as shown in Fig. 3.1A, which are a special kind of Recurrent neural network $(\underline{\mathrm{RNN}}$, capable of learning long-term dependencies. All RNNs have the form of a chain of repeating modules of neural networks. Similar structure with RNNs, but LSTM mechanism used four different interacting steps within each module: 1) Forget gate layer, using a sigmoid layer to decide what information the current module should throw away; 2) input gate layer, using a sigmoid and tanh layer to decide what new information the current module should store; 3) update the current module base on the first two steps; 4) output layer, using a sigmoid and tanh layer to decide what the current module should output. With this mechanism, the CNN+LSTM modality can capture temporal sequence and long-range information.

However, LSTMs on the last convolutional layers can have a large receptive field but may not 
A

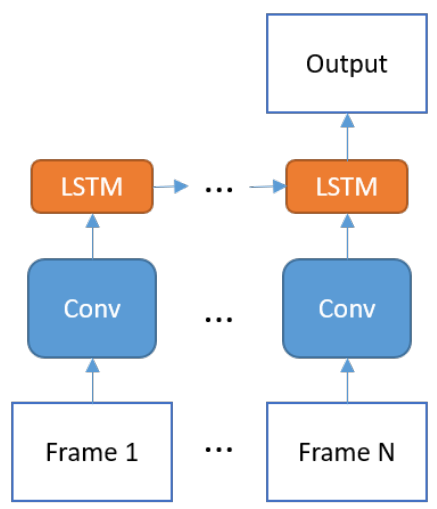

B

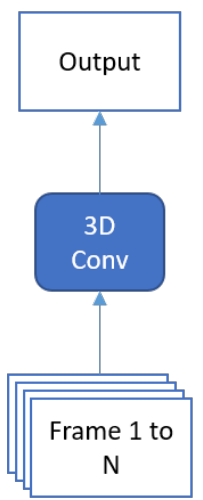

C

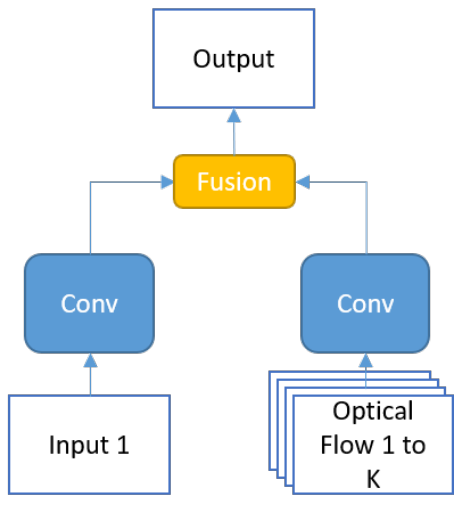

Figure 3.1: The main modalities in human action recognition area. (A) CNN+LSTM; (B) 3D CNN; (3) Two-Stream Network. N indicates for the total number of frames in a video, whereas $\mathrm{K}$ stands for a subset of neighboring frames of the video..

be able to capture fine low-level motion which is critical in many cases. It is also cost a lot of computing resource to unroll the network through multiple frames for backpropagation-throughtime.

\subsubsection{D CNN}

Another modality to model human actions is applied the 3D filter to the CNNs [16,17], as shown in Fig. 3.1B. They seem like the standard CNN, but with the spatial-temporal kernel which directly aggregated hierarchical representations of spatial-temporal data.

However, the image-based action recognition model is sensitive to a noisy background with complex occlusion or illumination conditions, changing camera view angles. The ability of the classification of this framework was also heavily dependent on the object in the images. For example, when there is a horse in the image, the model usually tends to mistakenly classify the action as horse riding, which means the model focus more on the object instead of the essential action. Moreover, It is expensive to train a 3D CNN, since the 3D filter has many more parameters than the $2 \mathrm{D}$ one. 


\subsubsection{Two Stream Network}

The second modality is the two-stream framework [13-15], as shown in Fig. 3.1C. This framework can be separated into two streams: 1) spatial stream. Using a single RGB frame, this stream served as an image classification architecture, which can extract useful clues from the static appearance as some actions are strongly associated with particular objects. 2) Temporal stream. Using a stack of pre-computed optical flow frames, this stream focuses on tracking the motion through the temporal domain, which can explicitly describes the motion between video frames. Fusing the prediction from these two streams, the framework can calculate the final output. Moreover, the fusion methods have been explored in the work of Feichtenhofer et al. [56]. They proposed spatial fusion, such as max, concatenation, Conv and bilinear fusions, and temporal fusion, e.g. 3D pooling to improve the performance of the two-stream framework.

However, the 3D CNN modality has the same issue as we discuss in Sec. 3.1.2, which is sensitive to the noisy background and heavily dependent on the object.

\subsubsection{Skeleton-base GCN}

GCN architecture,different from CNNs only can learn non-Euclidean structure data(i.e. image), can be applied to most of the topology graph dataset. For the above reasons, the GCN architecture has been effectively applied to a number of areas and become active last several years, especially in the topic of human action recognition when its effectiveness is demonstrated by ST-GCN. In this model, every input is defined as an external sequence of physical connection graphs where skeleton joints and bones are defined as graph nodes and edges respectively. With this skeletonbase input, the model is better robust against occlusion, illumination, and viewpoint change.

However, the GCN aggregates information based on an unchanged kernel (graph), designed according to the physical connection, through all the layers. It means the kernel is static and only describes physical edges between neighboring nodes, thus, cannot capture dynamic spatial correlations properly. For example, when we are clapping or hugging, in the graph, two hands should have a strong dependency but there is no connection in physical-based graph. Furthermore, with the data going deeper layers, the feature has a larger receptive field but the static kernel cannot match this change. 
In our study, we applied the ST-GCN model to our 2D skeleton-base ASD behavior recognition task. And aiming at solving the above issue, we adopted attention mechanism to enable our model get a adaptive graph during training.

\subsection{Background}

\subsubsection{Spatial-temporal graph}

Every input is defined as an external sequence of physical connection graphs where skeleton joints and bones are defined as graph nodes and edges respectively. For the spatial domain, the nodes in the same frame are connected with edges based on the connectivity of human body structure. For the temporal domain, each node is connected to the joint in the prior and next frame. In the study, we use an adjacent matrix A to denote the relationship between each node.

\subsubsection{Human skeleton graph convolution}

Basically, skeleton-base $\mathrm{GCN}$ action recognition models [18, 57] are composed of several spatial-temporal GCN blocks. The block can be separated into two steps. First, the model uses graph convolution to aggregate spatial feature according to the adjacent matrix $\mathbf{A}$. Then a general convolution will extract the temporal information from the output data of the first step. Therefore, the graph convolution plays a crucial role in the module, which not only is the key to the model expression power of spatial feature, but also directly affect the performance of extracting temporal information.

Let define $\mathbf{X} \in \mathbf{R}^{n \times C}$ be the input features in current frame, and $\mathbf{X}^{\prime} \in \mathbf{R}^{n \times C^{\prime}}$ be the output features of the graph convolution, where $C$ and $C^{\prime}$ are the dimension of the input and output feature respectively. The spatial graph convolution can be written as:

$$
\mathbf{X}^{\prime}=\widetilde{\mathbf{A}} \mathbf{X} \mathbf{W}
$$

where $\mathbf{W} \in \mathbf{R}^{C \times C^{\prime}}$ is the trainable weigh. And $\mathbf{A} \in \hat{\mathbf{R}}^{n \times n}$ is the normalized $\mathbf{A}$, which can be calculated according to node degree by transforming the adjacency matrix $\mathbf{A}$ by multiplying it with the 
inverse degree matrix $\mathbf{D} \in \mathbf{R}^{n \times n}$, the formulation can be written as:

$$
\widetilde{\mathbf{A}}=\mathbf{D}^{-1} \mathbf{A} \text {. }
$$

\subsection{Datasets and Data Preprocessing}

\subsubsection{NTU RGB+D}

NTU RGB+D dataset [58] is currently the largest and most widely used dataset for the human action recognition task. This dataset contains 56,880 video samples and categorized into 60 classes, captured from 40 different human subjects. including single-person action (e.g. wipe fave, walk and run) and two-person interactions (e.g. shaking hands, take a photo, and walking towards). The dataset provides four different modalities of data: 3D skeletons sequence, depth map sequences, RGB videos, and infrared videos. In our study, we only focused on the 3D skeletons sequence. The skeleton dataset is a sequence of 3D spatial coordinates and joint detection confidence of 25 joints for each human in an action sample and shown in Fig. 3.2, left. It is concurrently captured by three Kinect V2 cameras from different horizontal angles: $-45^{\circ}, 0^{\circ}, 45^{\circ}$.

In addition, there are two evaluation metrics developed on this dataset: 1) Cross-View (CV) evaluation: samples from the $0^{\circ}$ and $45^{\circ}$ cameras are used for training, and samples from the $-45^{\circ}$ camera are used for testing. The training and testing sets have 37,920 and 18,960 samples, respectively. 2) Cross-Subject (CS) evaluation: 40 subjects are split into training and testing groups. Each group consists of 20 subjects. The training and testing sets have 40,320 and 16,560 samples, respectively. We used this dataset to evaluate our adaptive graph and report the top- 1 and top-5 recognition accuracy on both evaluations.

\subsubsection{ASD Demonstration Task Interview}

We propose the ADTI dataset for our video-based ASD behavior recognition task. The data is recorded from the ADOS-2 interview that includes 15 scenarios. In our study, we used only scenario 8 , where the specialist aims to test the demonstration capacity of participants by asking them to represent how they brush their teeth and wash their face. This dataset is categorized into positive (participants with ASD) and negative (peers without ASD), and contains 95 clips where 

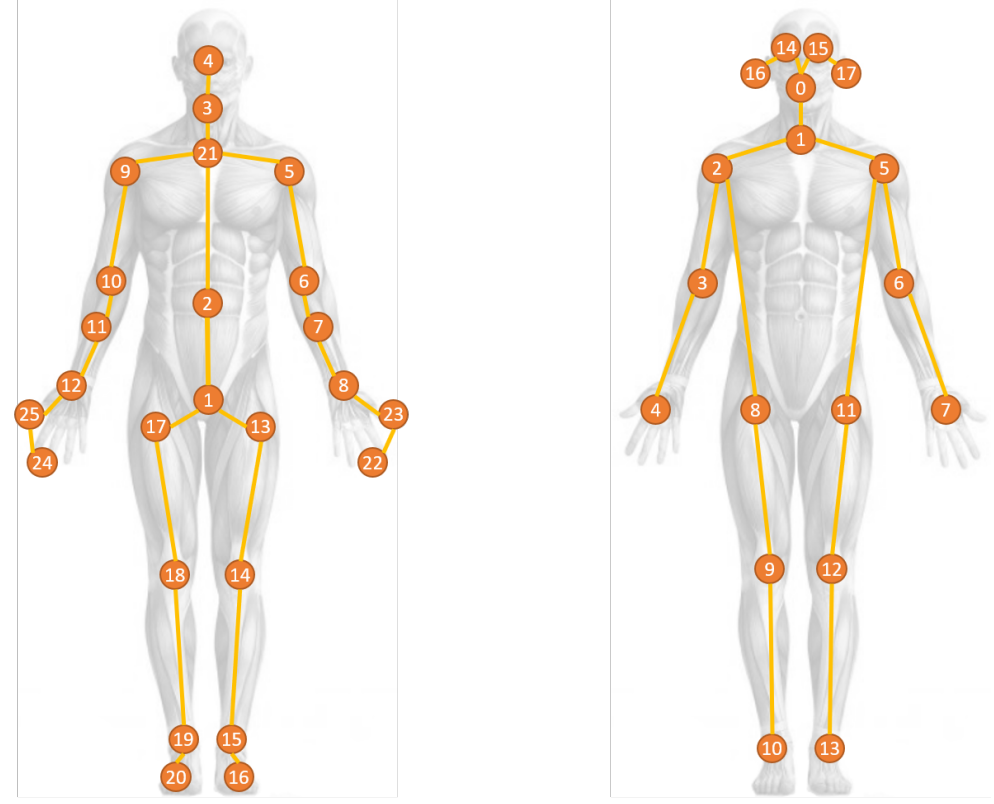

Figure 3.2: Illustration of the human skeleton graphs in NTU RGB+D dataset (left) and OpenPose post-detection skeleton (right).

37 clips are from the former and 58 clips are from the latter. Each clip is clipped from scenario 8 and contains from 150 to 300 frames. There is a total of 13 different human subjects participating in our interview, including 8 positive subjects and 5 negative subjects.

In the data preprocessing, firstly, we cropped the subject bounding box from each clip and resized them to the size of $224 \times 224$. Then to detect the human skeleton, we adopted the effective OpenPose framework [59-61] to our data. For each clip, the OpenPose can detect a sequence of 17-joint-skeleton, where each joint feature consists of 2D spatial coordinate and joint detection confidence. The OpenPose post-detection skeleton is shown in Fig. 3.2, right.

In our experiment, we applied the ST-GCN to this $2 \mathrm{D}$ skeleton data for our video-based ASD behavior recognition task. And a same CS evaluation with NTU RGB-D was adopted but the training and testing sets were separated by 5 -fold cross-validation strategy. 


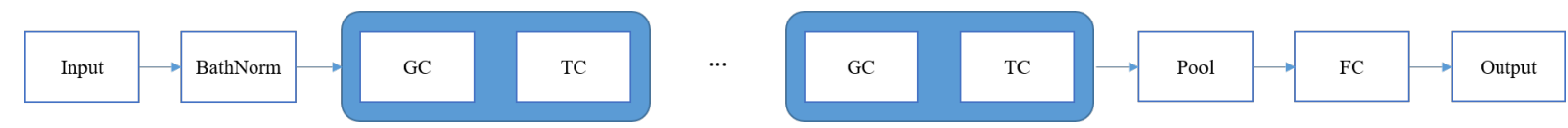

Figure 3.3: The input will be feed into a BatchNorm layer and nine blocks of spatial-temporal graph convolution (ST-GCN) will be applied and gradually generate higher-level feature maps on the graph. It will then be classified by the standard Softmax classifier to the corresponding action category.

\subsection{Methods and Modules of Adaptive Graph}

\subsubsection{Model Architecture}

We have applied the ST-GCN as our baseline, shown in Fig. 3.3. This GCN is capable of recognizing a large class of human actions [18], including the same action, wipe face and brush teeth, as our Autism Demonstration Task Interview (ADTI) dataset, and is thus suitable for our ASD behavior recognition task. The GCN compose of a feature extraction section (one BatchNorm layer and nine ST-GCN blocks) and a classification section (one FClayers).

In the first layer of the feature extraction section, the input data is fed into a BatchNorm, which can make the model converge smoothly and efficiently due to the position of a joint change a lot along with the temporal domain. And then the normalized data would be aggregated in nine ST-GCN blocks. The ST-GCN can be separated into two steps: 1) aggregating spatial feature (Graph Convolution $(\overline{G C})$ ). We applied a $3 \times 1$ filter with a stride of 1 to the graph convolution to aggregate spatial feature based on the topology graph (adjacent matrix). Especially, in block 4 and block 7, instead of using a stride of 1, a stride of 2 is applied to serve as a pooling layer. 2) aggregating temporal feature (Temporal Convolution (TC)). We used a $9 \times 1$ filter with a stride of 1 to the general convolution to aggregate the temporal information from the output data of the GC. In each TC, a BatchNorm and a ReLU operation were applied before the convolutional layer, which is followed by a BatchNorm and 50\% dropout to avoid overfitting. At the end of each ST-GCN block, a ReLU and a ResNet mechanism are adopted to raise the function of avoiding overfitting.

There were one global pooling and one FC layer in each classification section. With the spatialtemporal feature aggregated from the feature extraction section, each sequence would get a dimension of 256 feature vectors through the global pooling layer. Finally, an FC layer was used, which has the same number of channels as the number of classes (60 classes in NTU RGB+D dataset 
and two classes in ADTI), and a nonlinear Softmax operation was applied to the final output of the GCN to make the classification prediction. It is worth noting that the classification was performed for each task condition separately.

\subsubsection{Adaptive Graph}

The GCN aggregates information based on an unchanged kernel (graph), designed according to the physical connection, through all the layers. This kernel not only cannot represent the connection for most of the actions but also cannot match the variation of the receptive field when data are going deeper layers. Therefore, we suggested that this kernel may be not suitable for action recognition. To improve the performance of $\mathrm{GCN}$, the traditional $\mathrm{GCN}$ needs a dynamic kernel and a different graph for each ST-GCN block.

We adopted an adaptive graph mechanism, essentially an attention mechanism, to dynamically learn an exclusive adjacent matrix for each ST-GCN block. It is worth noting that the graph is described as adjacent matrix $\mathbf{A}$ and the attention mechanism can be described as attention matrix $\mathbf{B} \in \mathbf{R}^{n \times n}$. Let define $\mathbf{A}_{a d p} \in \mathbf{R}^{n \times n}$ be the adaptive adjacent matrix. In our experiment, we applied three different kinds of graphs for the graph convolution: 1) A. This is the original GCN kernel setting as the physical connection, which is inflexible during the training process. 2) $\mathbf{A}_{a d p}=\mathbf{A} \odot \mathbf{B}$, where the elements of the learnable $\mathbf{B}$ are initialized as 1. This kernel serves as an attention mask to magnify or minify the importance between two joints. Nevertheless, the multiplication operation cannot change the elements that are 0 in the original adjacency matrix, which means this kernel can change only the strength of existing edges and cannot add a new edge. 3) $\mathbf{A}_{a d p}=\mathbf{A}+\mathbf{B}$, where the elements of the learnable $\mathbf{B}$ are initialized as 0 . This kernel serves as an auxiliary adjacent matrix to increase or decrease the strength between two joints. Due to the addition operation, if necessary, the model can add useful edges or remove unimportant edges.

Especially, during the training process, the last two kinds of kernels would be normalized by Eqn. 3.2 to help the model converge smoothly and efficiently. Furthermore, to avoid the adaptive graph dramatically fluctuate and reduce the efficiency of model convergence at the beginning of the training process, we froze $\mathbf{B}$ at the first epoch to use physical connection $\mathbf{A}$ to initialize the parameter of the model. 


\subsection{Experimental Results}

In this section, we demonstrate the network setting, training details, ablation study and experimental results of proposed JDSR problem.

\subsubsection{Implementation Details}

In our model, all of the models are trained with the same batch size (64), learning schedule (SGD with an initial learning rate as 0.1 and reduced by 10 in epoch 20, 40, and 60), random seed (42), and training epochs (80) with Pytorch [43] deep learning framework. We used Nesterov momentum of 0.9 and weight decay of 0.0001 . Cross-entropy is selected as the loss function to backpropagate gradients.

We applied a $3 \times 1$ filter with a stride of 1 to most of the GC to aggregate spatial feature based on the adjacent matrix. Especially, in block 4 and block 7, instead of using a stride of 1, a stride of 2 is applied to serve as a pooling layer. A $9 \times 1$ filter with a stride of 1 and a padding of 4 is applied to each TC to aggregate the temporal information from the output data of the GC. The output channel of ST-GCN blocks 1-3 is set to 64, the output channel of ST-GCN blocks 4-6 is set to 128 , and the output channel of ST-GCN blocks 7-9 is set to 256 .

For the NTU RGB+D dataset, there are at most two people in each sample of the dataset. If the number of bodies in the sample is less than 2 , we pad the second body with 0 . The max number of frames in each sample is 300. For samples with less than 300 frames, we repeat the samples until it reaches 300 frames.

For the ADTI dataset, there are at most one people in each sample of the dataset. We perform the same data-augmentation methods as in [18]. In detail, we randomly choose 150 frames from the input skeleton sequence and slightly disturb the joint coordinates with randomly chosen rotations and translations.

\subsubsection{Ablation Study}

In this section, firstly, we demonstrate the effectiveness and efficiency of our adaptive graph mechanism on NTU RGB+D. And then we examine whether the ST-GCN baseline with our adap- 


\begin{tabular}{l|c|c|c|c}
\hline \multirow{2}{*}{ Kernel } & \multicolumn{2}{|c|}{ CV } & \multicolumn{2}{c}{ CS } \\
\cline { 2 - 5 } & Top-1(\%) & Top-5(\%) & Top-1(\%) & Top-5(\%) \\
\hline \hline $\mathbf{A}$ & 85.78 & 98.28 & 68.53 & 92.89 \\
$\mathbf{A} \odot \mathbf{B}$ & 88.35 & $\mathbf{9 8 . 8 3}$ & 81.98 & 97.25 \\
$\mathbf{A}+\mathbf{B}$ & $\mathbf{8 9 . 0 6}$ & 98.57 & $\mathbf{8 2 . 7 6}$ & $\mathbf{9 7 . 5 6}$ \\
\hline
\end{tabular}

Table 3.1: Performance of three different kernel on NTU RGB+D dataset. Bold font indicates the best result.

tive graph mechanism can be applied to ASD behavior recognition task on the ADTI dataset.

\section{Adaptive Graph}

First, we evaluate the necessity of applying the adaptive graph. Tab. 3.1 shows the results. To prove up to the hilt that our adaptive graph can improve the GCN, we evaluate our model on a large-scale dataset, NTU RGB+D. A indicates the original $\mathrm{GCN}$ kernel setting as the physical connection. $\mathbf{A} \odot \mathbf{B}$ indicates the multiplication operation approach, which cannot change the elements that are zero in the original adjacency matrix, which means this kernel can change only the strength of existing edges and cannot add a new edge. This approach outperforms the original unchanged kernel at $2.57 \%$ top- 1 and $0.55 \%$ top-5 on CV evaluation, and $13.45 \%$ top- 1 and $4.36 \%$ top-5 on CS evaluation. $\mathbf{A}+\mathbf{B}$ indicates the addition operation approach, which can add useful edges or remove unimportant edges if necessary. This approach outperforms the original unchanged kernel at $3.28 \%$ top- 1 and $0.29 \%$ top-5 on $\mathrm{CV}$ evaluation, and $14.23 \%$ top- 1 and $4.67 \%$ top-5 on CS evaluation. Therefore, the superiority of the adaptive graph, which flexibly describes the useful connection of joints, is verified.

\section{Application of GCN on 2D Skeleton-based ASD Behavior Recognition}

Because of the original ST-GCN designs for a 3D skeleton-based dataset. In this experiment, we examined the application of the ST-GCN with an adaptive graph in our 2D skeleton-based ADTI dataset. Tab. 3.2 shows the results. To get a stable performance of our model, we adopted a 5-fold cross-validation strategy. The model reached an accuracy of $72.5 \% \pm 23.07 \%$ for the original unchanged graph, $77.39 \% \pm 15.53 \%$ for the multiplicative adaptive graph, and $80.76 \% \pm$ 


\begin{tabular}{l|c|c|c}
\hline \multirow{2}{*}{ Fold Id } & \multicolumn{3}{|c}{ CS } \\
\cline { 2 - 4 } & $\mathbf{A}(\%)$ & $\mathbf{A} \odot \mathbf{B}(\%)$ & $\mathbf{A}+\mathbf{B}(\%)$ \\
\hline \hline 1 & 100.00 & 100.00 & 100.00 \\
2 & 74.19 & 77.42 & 77.42 \\
3 & 69.57 & 78.26 & 82.61 \\
4 & 43.75 & 56.25 & 68.75 \\
5 & 75.00 & 75.00 & 75.00 \\
\hline \hline Average & 72.50 & 77.386 & 80.76 \\
\hline
\end{tabular}

Table 3.2: Performance of the application of the ST-GCN with three different graph connection approaches in our 2D skeleton-based ADTI dataset.

$11.86 \%$ for the additive adaptive graph. The performance of the original graph shows that the STGCN can be successfully applied to the 2D skeleton-based behavior recognition task. Furthermore, the performance of the multiplicative and additive adaptive graph further proves that the adaptive graph mechanism can improve the effectiveness of GCN It is worth noting that the result of the additive adaptive graph have more effective and stabler than the other approaches because this method allows the model to add or remove edges during the training process.

\subsection{Summary}

In this chapter, we proposed the application of ST-GCN to ASD behavior recognition task. The proposed model adopted an adaptive graph mechanism that can help the model learn a flexible and exclusive topology graph for each ST-GCN block to match the variation of the receptive field when the layer getting deeper. And we demonstrated the necessity of an adaptive graph mechanism on NTU RGB+D and ADTI datasets. Finally, we showed the effectiveness of the ST-GCN application to our 2D skeleton-based task. 


\section{Chapter 4}

\section{Conclusions and Future Work}

In this dissertation, we have mainly studied two kinds of ASD diagnosis methods: 1) analysis of ASD first-person perspective, which demonstrated the effectiveness of the application of VGG16; 2) ASD behavior recognition, which shows the effectiveness of the application of ST-GCN.

\subsection{Analysis of ASD First-Person Perspective}

In this work, we applied a fine-tuning VGG16 to classify photos taken in three different categories (people, indoors, and outdoors) as either having been taken by individuals with ASD or by peers without $\mathrm{ASD}$, Our classifier effectively discriminated photos from all three categories but was particularly successful at classifying photos of people with $>80 \%$ accuracy. Importantly, the visualization of our model revealed critical features that led to successful discrimination and showed that our model adopted a strategy similar to that of ASD experts. Furthermore, for the first time, we showed that photos taken by individuals with ASD contained less salient objects, especially in the central visual field. Notably, our model outperformed the classification of these photos by ASD experts. Together, we demonstrate an effective and novel method that is capable of discerning photos taken by individuals with ASD and revealing aberrant visual attention in ASD from a unique first-person perspective. Our method may in turn provide an objective measure for evaluations of individuals with ASD. 


\subsection{ASD Behavior Recognition}

In this work, we applied a ST-GCN to classify clips recording the brush teeth and wipe face actions as either from participants with ASD or by peers without ASD. Our classifier effectively achieve a fine performance on our ADTI dataset. To further improve our model, we introduced a adaptive graph mechanism to learn a exclusive adaptive kernel for different ST-GCN blocks. From the results, our $\mathbf{A} \odot \mathbf{B}$ approach outperformed the baseline at $2.57 \%$ top-1 and $0.55 \%$ top-5 on CV evaluation, and $13.45 \%$ top-1 and $4.36 \%$ top-5 on CS evaluation. Meanwhile, our $\mathbf{A}+\mathbf{B}$ outperformed the original unchanged kernel at 3.28\% top-1 and $0.29 \%$ top-5 on CV evaluation, and $14.23 \%$ top- 1 and $4.67 \%$ top-5 on CS evaluation. It show that the adaptive graph mechanism plays an important role in improving the performance.

\subsection{Future Works}

Although in Chapter 3, we proposed an adaptive graph mechanism to improve the ST-GCN performance, the accuracy still needs to further improve and we need an explainable method to visualize how the model work. In future work, we will mainly focus on applying $\mathrm{GCN}$ to the video-based ASD behavior recognition area. Here, we discuss the future works.

First, apply a diversity graph to our model. The general CNNs have several kernels in the convolution process, which help the model learn different kinds of feature maps and the model would learn which features are crucial for the classification. In future work, we will explore a diversity graph mechanism to allow the $\mathrm{GCN}$ to extract more and diverse feature maps.

Furthermore, learn an exclusive graph for each class. In fact, different action requires a different topology graph. For example, when we are walking, the connection between hands and feet should be stronger, while the edge between hands and face should be stronger when we are wiping our face. With this approach, we also can visualize the graph of individuals with ASD and peers without ASD to find out the distinction between these two classes.

Finally, the ASD behavior recognition method should be ultimately applied to the Children dataset. According to the statistics, the first signs of ASD often visible by one year of age, while the average age of diagnosis is over four years old in the U.S. It is getting difficult to cure an 
individual with ASD with the age growing. Therefore, we should ultimately focus our method on children's ASD diagnosis. 


\section{Bibliography}

[1] M. Ruan, P. J. Webster, X. Li, and S. Wang, "Deep neural network reveals the world of autism from a first-person perspective," Autism Research, 2020.

[2] S. Wang and R. Adolphs, "Social saliency," in Computational and cognitive neuroscience of vision, pp. 171-193, Springer, 2017.

[3] S. Wang, M. Jiang, X. M. Duchesne, E. A. Laugeson, D. P. Kennedy, R. Adolphs, and Q. Zhao, "Atypical visual saliency in autism spectrum disorder quantified through model-based eye tracking," Neuron, vol. 88, no. 3, pp. 604-616, 2015.

[4] S. Wang, J. Xu, M. Jiang, Q. Zhao, R. Hurlemann, and R. Adolphs, "Autism spectrum disorder, but not amygdala lesions, impairs social attention in visual search," Neuropsychologia, vol. 63, pp. 259-274, 2014.

[5] A. Klin, W. Jones, R. Schultz, F. Volkmar, and D. Cohen, "Visual fixation patterns during viewing of naturalistic social situations as predictors of social competence in individuals with autism," Archives of general psychiatry, vol. 59, no. 9, pp. 809-816, 2002.

[6] K. A. Pelphrey, N. J. Sasson, J. S. Reznick, G. Paul, B. D. Goldman, and J. Piven, "Visual scanning of faces in autism," Journal of autism and developmental disorders, vol. 32, no. 4, pp. 249-261, 2002.

[7] G. Dawson, S. J. Webb, and J. McPartland, "Understanding the nature of face processing impairment in autism: insights from behavioral and electrophysiological studies," Developmental neuropsychology, vol. 27, no. 3, pp. 403-424, 2005.

[8] N. J. Sasson, J. T. Elison, L. M. Turner-Brown, G. S. Dichter, and J. W. Bodfish, "Brief report: Circumscribed attention in young children with autism," Journal of autism and developmental disorders, vol. 41, no. 2, pp. 242-247, 2011.

[9] L. Kanner et al., "Autistic disturbances of affective contact," Nervous child, vol. 2, no. 3, pp. 217-250, 1943.

[10] A. Le Couteur, M. Rutter, C. Lord, P. Rios, S. Robertson, M. Holdgrafer, and J. McLennan, "Autism diagnostic interview: a standardized investigator-based instrument," Journal of autism and developmental disorders, vol. 19, no. 3, pp. 363-387, 1989. 
[11] J. Donahue, L. Anne Hendricks, S. Guadarrama, M. Rohrbach, S. Venugopalan, K. Saenko, and T. Darrell, "Long-term recurrent convolutional networks for visual recognition and description," in Proceedings of the IEEE conference on computer vision and pattern recognition, pp. 2625-2634, 2015.

[12] J. Yue-Hei Ng, M. Hausknecht, S. Vijayanarasimhan, O. Vinyals, R. Monga, and G. Toderici, "Beyond short snippets: Deep networks for video classification," in Proceedings of the IEEE conference on computer vision and pattern recognition, pp. 4694-4702, 2015.

[13] K. Simonyan and A. Zisserman, "Two-stream convolutional networks for action recognition in videos," in Advances in neural information processing systems, pp. 568-576, 2014.

[14] L. Wang, Y. Xiong, Z. Wang, Y. Qiao, D. Lin, X. Tang, and L. Van Gool, “Temporal segment networks: Towards good practices for deep action recognition," in European conference on computer vision, pp. 20-36, Springer, 2016.

[15] C. Feichtenhofer, H. Fan, J. Malik, and K. He, "Slowfast networks for video recognition," in Proceedings of the IEEE international conference on computer vision, pp. 6202-6211, 2019.

[16] D. Tran, L. Bourdev, R. Fergus, L. Torresani, and M. Paluri, "Learning spatiotemporal features with 3d convolutional networks," in Proceedings of the IEEE international conference on computer vision, pp. 4489-4497, 2015.

[17] D. Tran, H. Wang, L. Torresani, J. Ray, Y. LeCun, and M. Paluri, "A closer look at spatiotemporal convolutions for action recognition," in Proceedings of the IEEE conference on Computer Vision and Pattern Recognition, pp. 6450-6459, 2018.

[18] S. Yan, Y. Xiong, and D. Lin, "Spatial temporal graph convolutional networks for skeletonbased action recognition," arXiv preprint arXiv:1801.07455, 2018.

[19] C. Ames and S. Fletcher-Watson, "A review of methods in the study of attention in autism," Developmental Review, vol. 30, no. 1, pp. 52-73, 2010.

[20] E. Birmingham, M. Cerf, and R. Adolphs, "Comparing social attention in autism and amygdala lesions: effects of stimulus and task condition," Social neuroscience, vol. 6, no. 5-6, pp. 420-435, 2011.

[21] L. Byrge, J. Dubois, J. M. Tyszka, R. Adolphs, and D. P. Kennedy, "Idiosyncratic brain activation patterns are associated with poor social comprehension in autism," Journal of Neuroscience, vol. 35, no. 14, pp. 5837-5850, 2015.

[22] K. Chawarska, S. Macari, and F. Shic, "Decreased spontaneous attention to social scenes in 6-month-old infants later diagnosed with autism spectrum disorders," Biological psychiatry, vol. 74, no. 3, pp. 195-203, 2013.

[23] K. Rice, J. M. Moriuchi, W. Jones, and A. Klin, "Parsing heterogeneity in autism spectrum disorders: visual scanning of dynamic social scenes in school-aged children," Journal of the American Academy of Child \& Adolescent Psychiatry, vol. 51, no. 3, pp. 238-248, 2012. 
[24] A. Santos, T. Chaminade, D. Da Fonseca, C. Silva, D. Rosset, and C. Deruelle, "Just another social scene: evidence for decreased attention to negative social scenes in high-functioning autism," Journal of autism and developmental disorders, vol. 42, no. 9, pp. 1790-1798, 2012.

[25] F. Shic, J. Bradshaw, A. Klin, B. Scassellati, and K. Chawarska, "Limited activity monitoring in toddlers with autism spectrum disorder," Brain research, vol. 1380, pp. 246-254, 2011.

[26] M. Freeth, P. Chapman, D. Ropar, and P. Mitchell, "Do gaze cues in complex scenes capture and direct the attention of high functioning adolescents with asd? evidence from eye-tracking," Journal of autism and developmental disorders, vol. 40, no. 5, pp. 534-547, 2010.

[27] C. F. Norbury, J. Brock, L. Cragg, S. Einav, H. Griffiths, and K. Nation, "Eye-movement patterns are associated with communicative competence in autistic spectrum disorders," Journal of child psychology and psychiatry, vol. 50, no. 7, pp. 834-842, 2009.

[28] D. Riby and P. J. Hancock, "Looking at movies and cartoons: eye-tracking evidence from williams syndrome and autism," Journal of Intellectual Disability Research, vol. 53, no. 2, pp. 169-181, 2009.

[29] D. M. Riby, P. J. Hancock, N. Jones, and M. Hanley, "Spontaneous and cued gaze-following in autism and williams syndrome," Journal of neurodevelopmental disorders, vol. 5, no. 1, p. 13, 2013.

[30] E. Marinoiu, M. Zanfir, V. Olaru, and C. Sminchisescu, "3d human sensing, action and emotion recognition in robot assisted therapy of children with autism," in Proceedings of the IEEE Conference on Computer Vision and Pattern Recognition, pp. 2158-2167, 2018.

[31] J. Rehg, G. Abowd, A. Rozga, M. Romero, M. Clements, S. Sclaroff, I. Essa, O. Ousley, Y. Li, C. Kim, et al., "Decoding children's social behavior," in Proceedings of the IEEE conference on computer vision and pattern recognition, pp. 3414-3421, 2013.

[32] S. Wang, S. Fan, B. Chen, S. Hakimi, L. K. Paul, Q. Zhao, and R. Adolphs, "Revealing the world of autism through the lens of a camera," Current Biology, vol. 26, no. 20, pp. R909R910, 2016.

[33] M. Duda, J. Kosmicki, and D. Wall, "Testing the accuracy of an observation-based classifier for rapid detection of autism risk," Translational psychiatry, vol. 4, no. 8, pp. e424-e424, 2014.

[34] Q. Tariq, J. Daniels, J. N. Schwartz, P. Washington, H. Kalantarian, and D. P. Wall, "Mobile detection of autism through machine learning on home video: A development and prospective validation study," PLoS medicine, vol. 15, no. 11, p. e1002705, 2018.

[35] D. P. Wall, J. Kosmicki, T. Deluca, E. Harstad, and V. A. Fusaro, "Use of machine learning to shorten observation-based screening and diagnosis of autism," Translational psychiatry, vol. 2, no. 4, pp. e100-e100, 2012. 
[36] M. Jiang and Q. Zhao, "Learning visual attention to identify people with autism spectrum disorder," in Proceedings of the IEEE International Conference on Computer Vision, pp. 32673276, 2017.

[37] V. Hus and C. Lord, "The autism diagnostic observation schedule, module 4: revised algorithm and standardized severity scores," Journal of autism and developmental disorders, vol. 44, no. 8, pp. 1996-2012, 2014.

[38] A. Le Couteur, M. Rutter, C. Lord, P. Rios, S. Robertson, M. Holdgrafer, and J. McLennan, "Autism diagnostic interview: a standardized investigator-based instrument," Journal of autism and developmental disorders, vol. 19, no. 3, pp. 363-387, 1989.

[39] C. Lord, M. Rutter, and A. Le Couteur, "Autism diagnostic interview-revised: a revised version of a diagnostic interview for caregivers of individuals with possible pervasive developmental disorders," Journal of autism and developmental disorders, vol. 24, no. 5, pp. 659-685, 1994.

[40] M. Rutter, A. Bailey, and C. Lord, The social communication questionnaire: Manual. Western Psychological Services, 2003.

[41] K. Simonyan and A. Zisserman, "Very deep convolutional networks for large-scale image recognition," arXiv preprint arXiv:1409.1556, 2014.

[42] Q. Hou, M.-M. Cheng, X. Hu, A. Borji, Z. Tu, and P. H. Torr, "Deeply supervised salient object detection with short connections," in Proceedings of the IEEE Conference on Computer Vision and Pattern Recognition, pp. 3203-3212, 2017.

[43] A. Paszke, S. Gross, S. Chintala, G. Chanan, E. Yang, Z. DeVito, Z. Lin, A. Desmaison, L. Antiga, and A. Lerer, "Automatic differentiation in pytorch," in NIPS-W, 2017.

[44] V. Subramanian, Deep Learning with PyTorch: A practical approach to building neural network models using PyTorch. Packt Publishing Ltd, 2018.

[45]

[46] F. Happé, A. Ronald, and R. Plomin, "Time to give up on a single explanation for autism," Nature neuroscience, vol. 9, no. 10, pp. 1218-1220, 2006.

[47] D. Bone, M. S. Goodwin, M. P. Black, C.-C. Lee, K. Audhkhasi, and S. Narayanan, "Applying machine learning to facilitate autism diagnostics: pitfalls and promises," Journal of autism and developmental disorders, vol. 45, no. 5, pp. 1121-1136, 2015.

[48] A. G. Reece and C. M. Danforth, "Instagram photos reveal predictive markers of depression," EPJ Data Science, vol. 6, no. 1, pp. 1-12, 2017.

[49] J. I. Borjon, S. E. Schroer, S. Bambach, L. K. Slone, D. H. Abney, D. J. Crandall, and L. B. Smith, "A view of their own: Capturing the egocentric view of infants and toddlers with headmounted cameras," JoVE (Journal of Visualized Experiments), no. 140, p. e58445, 2018. 
[50] J. M. Franchak, K. S. Kretch, K. C. Soska, and K. E. Adolph, "Head-mounted eye tracking: A new method to describe infant looking," Child development, vol. 82, no. 6, pp. 1738-1750, 2011.

[51] S. Jayaraman, C. M. Fausey, and L. B. Smith, "The faces in infant-perspective scenes change over the first year of life," PloS one, vol. 10, no. 5, p. e0123780, 2015.

[52] C. Yu and L. B. Smith, "Joint attention without gaze following: Human infants and their parents coordinate visual attention to objects through eye-hand coordination," PloS one, vol. 8, no. 11, p. e79659, 2013.

[53] J. M. Franchak and K. E. Adolph, "Visually guided navigation: Head-mounted eye-tracking of natural locomotion in children and adults," Vision research, vol. 50, no. 24, pp. 2766-2774, 2010.

[54] M. L. Spezio, P.-Y. S. Huang, F. Castelli, and R. Adolphs, "Amygdala damage impairs eye contact during conversations with real people," Journal of Neuroscience, vol. 27, no. 15, pp. 3994-3997, 2007.

[55] J. Xie, L. Wang, P. Webster, Y. Yao, J. Sun, S. Wang, and H. Zhou, "A two-stream end-to-end deep learning network for recognizing atypical visual attention in autism spectrum disorder," arXiv preprint arXiv:1911.11393, 2019.

[56] C. Feichtenhofer, A. Pinz, and A. Zisserman, "Convolutional two-stream network fusion for video action recognition," in Proceedings of the IEEE conference on computer vision and pattern recognition, pp. 1933-1941, 2016.

[57] K. Cheng, Y. Zhang, C. Cao, L. Shi, J. Cheng, and H. Lu, "Decoupling gen with dropgraph module for skeleton-based action recognition,"

[58] A. Shahroudy, J. Liu, T.-T. Ng, and G. Wang, "Ntu rgb+ d: A large scale dataset for 3d human activity analysis," in Proceedings of the IEEE conference on computer vision and pattern recognition, pp. 1010-1019, 2016.

[59] Z. Cao, G. Hidalgo Martinez, T. Simon, S. Wei, and Y. A. Sheikh, "Openpose: Realtime multi-person $2 \mathrm{~d}$ pose estimation using part affinity fields," IEEE Transactions on Pattern Analysis and Machine Intelligence, 2019.

[60] Z. Cao, T. Simon, S.-E. Wei, and Y. Sheikh, "Realtime multi-person 2 d pose estimation using part affinity fields," in CVPR, 2017.

[61] S.-E. Wei, V. Ramakrishna, T. Kanade, and Y. Sheikh, "Convolutional pose machines," in CVPR, 2016. 


\section{Appendix A}

\section{Supporting Information}

Additional supporting information may be found online in the Supporting Information section at the end of the article.

\section{Table S1}

Participant characterization. ASD was evaluated using a DSM-5 diagnosis, the Autism Diagnostic Observation Schedule-2 (ADOS-2), ADI-R [39], and Social Communication Questionnaire (SCQ) [40]. The ADOS-2 was scored according to the latest algorithm and Calibrated Severity Scores (CSSs) were derived for exploratory correlation analyses [37]. The ADOS-2 is a structured interaction with an experimenter that is videotaped and scored by trained clinical staff, yielding scores on several scales. The ADOS-2 revised algorithm cutoff scores indicating an ASD diagnosis are 6 for Social Affect and 8 for Social Affect plus Restricted and Repetitive Behavior. Calibrated severity scores for each domain range from 1 to 10 , with 10 indicating the greatest severity. ADOS item scores were not available for three ASD participants, so we were unable to utilize the revised scoring system; however, original ADOS-2 algorithm scores for these three participants are as follows: A4: Communication $=4$, Reciprocal Social Interaction $(\underline{\mathrm{RSI}})=9$, Imagination/Creativity $(\underline{\mathrm{IC}})=1$, Stereotyped Behaviors Restricted Interests $(\underline{\mathrm{SBRI}})=1$; A6: Communication $=4, \overline{\mathrm{RSI}}=5, \underline{\mathrm{IC}}=0, \underline{\mathrm{SBRI}}=1 ; \mathrm{A} 10$ : Communication $=6, \underline{\mathrm{RSI}}=11, \underline{\mathrm{IC}}=1, \underline{\mathrm{SBRI}}=$ 0 . 